\title{
Wavelet-based ULF wave diagnosis of substorm expansion phase onset
}

Article

Published Version

Murphy, K. R., Rae, I. J., Mann, I. R., Milling, D. K., Watt, C. E. J., Ozeke, L., Frey, H. U., Angelopoulos, V. and Russell, C. T. (2009) Wavelet-based ULF wave diagnosis of substorm expansion phase onset. Journal of Geophysical Research, 114. A00C16. ISSN 0148-0227 doi: https://doi.org/10.1029/2008JA013548 Available at https://centaur.reading.ac.uk/32824/

It is advisable to refer to the publisher's version if you intend to cite from the work. See Guidance on citing.

Published version at: http://dx.doi.org/10.1029/2008JA013548

To link to this article DOI: http://dx.doi.org/10.1029/2008JA013548

Publisher: American Geophysical Union

All outputs in CentAUR are protected by Intellectual Property Rights law, including copyright law. Copyright and IPR is retained by the creators or other copyright holders. Terms and conditions for use of this material are defined in the End User Agreement.

\section{www.reading.ac.uk/centaur}

\section{CentAUR}


Central Archive at the University of Reading

Reading's research outputs online 


\title{
Wavelet-based ULF wave diagnosis of substorm expansion phase onset
}

\author{
Kyle R. Murphy, ${ }^{1}$ I. Jonathan Rae, ${ }^{1}$ Ian R. Mann, ${ }^{1}$ David K. Milling, ${ }^{1}$ Clare E. J. Watt, ${ }^{1}$ \\ Louis Ozeke, ${ }^{1}$ Harald U. Frey, ${ }^{2}$ Vassilis Angelopoulos, ${ }^{3}$ and Christopher T. Russell ${ }^{3}$ \\ Received 27 June 2008; revised 23 September 2008; accepted 27 October 2008; published 13 February 2009.
}

[1] Using a discrete wavelet transform with a Meyer wavelet basis, we present a new quantitative algorithm for determining the onset time of Pi1 and Pi2 ULF waves in the nightside ionosphere with $\sim 20$ - to 40 -s resolution at substorm expansion phase onset. We validate the algorithm by comparing both the ULF wave onset time and location to the optical onset determined by the Imager for Magnetopause-to-Aurora Global Exploration (IMAGE)-Far Ultraviolet Imager (FUV) instrument. In each of the six events analyzed, five substorm onsets and one pseudobreakup, the ULF onset is observed prior to the global optical onset observed by IMAGE at a station closely conjugate to the optical onset. The observed ULF onset times expand both latitudinally and longitudinally away from an epicenter of ULF wave power in the ionosphere. We further discuss the utility of the algorithm for diagnosing pseudobreakups and the relationship of the ULF onset epicenter to the meridians of elements of the substorm current wedge. The importance of the technique for establishing the causal sequence of events at substorm onset, especially in support of the multisatellite Time History of Events and Macroscale Interactions During Substorms (THEMIS) mission, is also described.

Citation: Murphy, K. R., I. J. Rae, I. R. Mann, D. K. Milling, C. E. J. Watt, L. Ozeke, H. U. Frey, V. Angelopoulos, and C. T. Russell (2009), Wavelet-based ULF wave diagnosis of substorm expansion phase onset, J. Geophys. Res., 114, A00C16, doi:10.1029/2008JA013548.

\section{Introduction}

[2] Since the initial detailed characterization of the growth, expansion and recovery phases of substorms [Akasofu, 1964; McPherron, 1972], significant work has been dedicated to determining the mechanism (or mechanisms) responsible for the onset and subsequent evolution of substorms in the magnetosphere. Two models are currently favored to explain the observed phenomena associated with the expansion onset phase of a geomagnetic substorm: the Near Earth Neutral Line (NENL) [Russell and McPherron, 1973; Russell, 1974] (see also the review by Baker et al. [1996, and references therein]) and Current Disruption (CD) [e.g., Lui, 1996] models. In the NENL model, magnetic reconnection in the tail is responsible for the initiation of the expansion phase, while in the CD model, a plasma instability in the near-Earth plasma sheet initiates the substorm expansion phase.

[3] In the NENL model, reconnection in the magnetotail at distances $\sim 20-25 \operatorname{Re}$ [e.g., Baumjohann et al., 1989] drives Earthward flows in the form of bursty bulk flows (BBFs) [e.g., Angelopoulos et al., 1992]. These BBFs brake as they approach dipolar field lines closer to the Earth, disrupting the

\footnotetext{
${ }^{1}$ Department of Physics, University of Alberta, Edmonton, Alberta, Canada.

${ }^{2}$ Space Sciences Laboratory, University of California, Berkeley, California, USA.

${ }^{3}$ Institute of Geophysics and Planetary Physics, University of California, Los Angeles, California, USA.
}

Copyright 2009 by the American Geophysical Union. 0148-0227/09/2008JA013548 cross-tail current and diverting it into the ionosphere to form the Substorm Current Wedge (SCW) [e.g., Shiokawa et al., 1998]. In the CD model, plasma instabilities, such as ballooning modes [e.g., Roux et al., 1991], lower hybrid turbulence [e.g., Huba et al., 1977; Lui, 1996], or cross-field current instabilities [e.g., Lui et al., 1995] lead to diversion of the cross-tail current into the ionosphere forming the $\mathrm{SCW}$. In this model, rarefaction waves propagate outward and trigger reconnection at the NENL later in the expansion phase following the onset at the inner edge of the plasma sheet [e.g., Angelopoulos, 2008]. To date, the lack of sufficiently large-scale and sufficiently high spatial and temporal resolution of conjugate in situ and ground-based magnetic and auroral observations during the expansion phase of substorms has hindered efforts to resolve, both spatially and temporally, the sequence of events to unequivocally determine whether substorms onset occurs inside-toout (e.g., CD) or outside-to-in (e.g., NENL).

[4] Vital to characterizing the sequence of events observed during the evolution of the substorm is an accurate determination of the spatial and temporal onset of the substorm expansion phase. Traditionally the structure and dynamics of auroral arcs preceding the formation of the substorm current wedge have been used to determine the onset of the substorm expansion phase. Akasofu [1964] characterized the onset of the expansion phase as the brightening of a quiescent arc, "usually the most equatorward arc," followed by the rapid poleward expansion of the auroral arc. During periods of clears skies, ground-based optical instruments provide a characterization of the energetic particle precipitation during 
the expansion phase onset of substorms. However, we will show in this article that we can achieve a better understanding of the evolution of substorms by including ground-based observations of magnetic fluctuations, especially since disturbances with no optical counterpart (i.e., missing images or obscure by thick clouds) can then also be characterized.

[5] Substorms manifest themselves in ground-based magnetometers as large amplitude perturbations of the background field [Kisabeth and Rostoker, 1977] as the magnetotail dipolarizes and the SCW wedge forms in the magnetosphere and ionosphere. Prior to the full formation of the SCW, impulsive ULF waves denoted Pils and Pi2s with periods of $1-40 \mathrm{~s}$ and $40-150 \mathrm{~s}$, respectively, are observed [Jacobs et al., 1964]. Pi2s observed at substorm onset are believed to be generated by the initial disturbance of the magnetospheric plasma sheet during the expansion phase onset, and the propagation to the ionosphere and subsequent perturbations establish the field-aligned current (FAC) system in the SCW [Olson, 1999]. Though evidence suggests that these waves are intimately connected with the onset of the substorm expansion phase, the onset of Pi2 wave packets can generally be timed with a resolution of approximately the wave period $(\sim 1-2 \mathrm{~min})$. Pi2 waves are also commonly observed across many hours of local time, and hence their mesoscale occurrence cannot be used to identify any localized ionospheric region associated with substorm initiation. Note however that the polarization of Pi2s can be used to identify the meridians of the elements of the SCW [e.g., Lester et al., 1983]. Pils on the other hand have significantly shorter periods allowing for more accurate timing of substorm expansion phase onset.

[6] Initial studies of Pils observed at substorm onset concentrated on broadband ULF waves with periods of 1$10 \mathrm{~s}$, referred to as Pi1B waves [e.g., Bosinger et al., 1981; Lessard et al., 2006]. Posch et al. [2007] found that the onset of Pi1Bs are coincident in both time and space to the optical onset determined by the Imager for Magnetopause-to-Aurora Global Exploration (IMAGE)-Far Ultraviolet Imager (FUV). These authors concluded that a very dense array of searchcoil magnetometers may provide improved information and resolution of the ionospheric onset location. Milling et al. [2008] have recently shown using discrete wavelet transform (DWT) techniques that long-period Pi1s can be used to define a ULF onset time to within a temporal resolution of $\sim 20 \mathrm{~s}$. These authors have shown that longperiod Pi1 waves, which are more readily studied with the current deployment of fluxgate magnetometers in the North American auroral zone then their shorter-period PilB counterparts, also have a localized ionospheric epicenter where the onset disturbance begins, which subsequently expands over continent scales.

[7] In this article we present further application of the newly developed DWT algorithm to examine the ionospheric onset of magnetic substorms. The DWT algorithm and results are validated by comparing optical onsets determined by the IMAGE-FUV instrument, with a $2 \mathrm{~min}$ cadence, on board the IMAGE satellite, to the Pi1/2 ULF onset time and ionospheric location determined using the DWT algorithm. We present here five events identified in the Frey substorm database [Frey et al., 2004; Frey and Mende, 2006] and in each of the substorm events we find a coherent expansion of ULF waves away from an epicenter of ULF power, consistent with the Pi1/2 expansion observed by Milling et al. [2008]. The Pi1/2 ULF DWT algorithm hence represents a powerful new tool for characterizing the onset and evolution of substorms especially when combined with in situ satellite data, for example, Time History of Events and Macroscale Interactions During Substorms (THEMIS), and contributes to the resolution of the so-called 2-min problem [e.g., Angelopoulos, 2008, Tables 2 and 3; Petrukovich and Yahnin, 2006]. The ability to accurately determine timing, and hence the causal sequence of events in relation to the proposed $\mathrm{CD}$ and NENL hypotheses for substorm expansion phase onset, is key to ultimately solving the substorm problem.

\section{Automated Wavelet Estimation of Substorm Onset and Magnetic Events}

[8] Wavelets provide a novel tool for studying localized characteristics of a nonstationary series in both the frequency and time domains. Specifically the discrete wavelet transform (DWT) utilizes a complete wavelet basis (e.g., the Meyer [1989] or Daubechies [1988] wavelets) to decompose a signal into wavelet coefficients denoted by, $\alpha_{j, k}$, which are localized in time $(k)$ and band limited in frequency or period $(j)$. With the use of the DWT and a specified wavelet basis, the dispersive and impulsive characteristics of wave phenomena are more accurately represented than with the traditional Fourier transform.

[9] The DWT's ability to characterize both the frequency and temporal content of waves makes it ideal for studying Pi1 and Pi2 ULF waves during the onset of magnetic substorms. Nose et al. [1998] showed that the Meyer wavelet can be used for studying long-period and impulsive Pi2 ULF waves associated with substorm onset. These authors successfully developed an automated algorithm, utilizing a wavelet power threshold, for now-casting magnetic substorms from real-time midlatitude ground-based magnetometer data. For reference, Figure 1 shows the characteristic Meyer wavelet from the three unitary wavelet coefficients, $\alpha_{6,7}, \alpha_{5,7}$ and $\alpha_{4,7}$ (Figures 1a-1c, respectively). Table 1 shows the period band $(j)$, ULF band, temporal width $(k)$ and the associated timing uncertainty for a wavelet of specific $\mathrm{j}$. The period bands shown in Table 1 have values associated with the impulsive Pi1 and Pi2 ULF wave bands.

[10] The Automated Wavelet Estimation of Substorm Onset and Magnetic Events (AWESOME) technique presented here utilizes the Meyer wavelet to determine the onset time and characteristics of ULF waves as a function of $j$ observed by ground-based magnetometers. The AWESOME algorithm is applied to magnetometer data provided by the Canadian Array for Realtime Investigations of Magnetic Activity (CARISMA) [Mann et al., 2008], THEMIS [Russell et al., 2008], Canadian Magnetic Observatory System (CANMOS), Geophysical Institute Magnetometer Array (GIMA), and Midcontinent Magnetoseismic Chain (McMAC) magnetometer arrays (Figure 2) to determine the initiation time of ULF waves in the Pi1 and Pi2 bands in the North American sector following substorm expansion phase onset. Using the AWESOME algorithm and the extensive spatial coverage provided by these five magnetometer arrays provides a means to determine the spatial dependence of the onset time of Pi1 and Pi2 ULF waves at the onset of a 


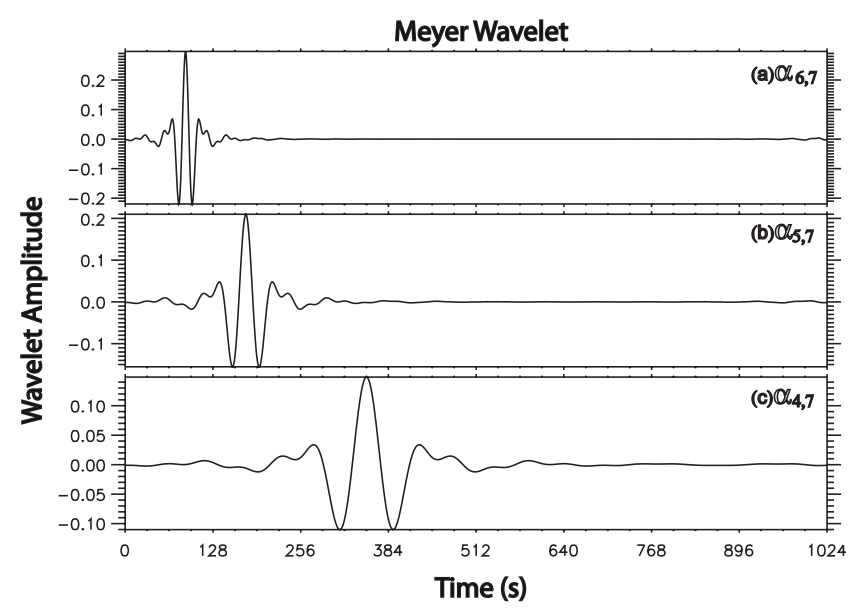

Figure 1. Select Meyer wavelets, $(j, k)$, representative of the Pi1 and Pi2 ULF waves observed during the expansion phase onset: (a) (6, 7)-Pi1/2; (b) (5, 7)-Pi1/2; (c) (4, 7)-Pi2.

magnetospheric substorm on a continental scale. The resulting expansion of these ULF wave signals in the ionosphere also indicates the capabilities of the AWESOME technique to identify an ionospheric epicenter of ULF wave activity using DWT methods [cf. Milling et al., 2008].

[11] It is sometimes difficult to estimate the onset of substorm-related ULF wave activity since the time series are noisy and contain fluctuations due to preexisting ionospheric currents. The AWESOME algorithm implements an interactive adaptive threshold to determine the time at which the signal at each magnetometer station in each $j$ band develops power above the preexisting noise. For each magnetometer station a period of low-amplitude ULF wave power before the substorm is selected. In all of the events we select the quietest interval preceding the substorm during the UT day, usually an interval greater than $5 \mathrm{~h}$, and calculate a noise threshold for each band based upon the properties of the waves during this time. While this is the case for each of the events presented herein, a quiet time preceding substorm onset cannot always be identified. In such cases other times, either earlier or later, could be selected to define magnetic quiet at each station and hence the appropriate thresholds. The calculation of a statistical noise threshold based upon prior quiet-time magnetometer data at each station allows the AWESOME algorithm to more objectively and quantitatively determine the onset time of substorm related ULF waves. The Meyer DWT power spectrum is calculated using the geomagnetic northsouth and east-west magnetic field components $(\mathrm{H}$ and $\mathrm{D}$, respectively), and the transverse wavelet power coefficients $\alpha_{j, k}$ are determined from the square root of the sum of squares of $\alpha_{j, k}^{H}$ and $\alpha_{j, k}^{D}$; i.e.,

$$
\alpha^{2}=\left(\left(\alpha^{D}\right)^{2}+\left(\alpha^{H}\right)^{2}\right)^{1 / 2} .
$$

The threshold power value for each wavelet frequency band, $j$, is defined as the mean plus two standard deviations of the quiet time wavelet power coefficients in that band. In the DWT applied here we use a window of $512 \mathrm{~s}$ of data with 1-s cadence. Since the DWT Meyer wavelet, like all mother wavelets, has length inversely proportional to $j$, we obtain more power estimates for large $j$ within the 512-s window. For example for the $j=4$, the wavelet is $64 \mathrm{~s}$ in length, generating a set of eight wavelet power coefficients to define the mean and standard deviation $(\sigma)$ of the $\mathrm{j}=4$ wavelet band during the 512-s window; for $j=9$, the wavelets are $2 \mathrm{~s}$ long, hence there are 256 estimates of wavelet power in the 512-s window with which to define the mean and standard deviation of the power for the $j=9$ wavelet band. This $2 \sigma$ threshold provides a 98\% confidence level that wavelet power coefficients above these thresholds represent a statistically significant signal and are not background noise. Note that in theory different threshold definitions can be used, this can be important for analyzing multiple or compound substorm intervals (i.e., nonisolated activations which evolve as a series of precursors or pseudobreakups followed by a main onset, often with subsequent intensifications) where power fluctuations do not return to presubstorm levels between multiple onsets. However, for isolated substorms we have determined that in practice two standard deviations represent a good threshold. The time of onset of substorm-related ULF activity is defined by using the first $j$ band to exhibit a continuous time series of transverse wavelet coefficients whose power exceeds the threshold value. The onset time is then defined to be at the center of the first wavelet coefficient which exceeds the threshold, the uncertainty being defined as plus or minus half the temporal width of each coefficient for the specific onset band (compare Table 1).

\section{Observations}

[12] The Frey substorm database contains the onset times and locations for substorm onsets identified by the FUV imager, at a cadence of $2 \mathrm{~min}$, onboard the IMAGE satellite between May 2000 and December 2005 [Frey et al., 2004]. These onset times were identified as the frame showing the first evidence of auroral intensification, the onset location being described as the brightest pixel in the frame. Highresolution, 1-s cadence, GPS-timed CARISMA magnetometer data was available for the entire upgraded CARISMA array from 1 April 2005. In order to establish the relationship between the timing and spatial location of AWESOME onsets with those in the Frey database, we chose to examine onsets between 1 April 2005 and the end of December 2005. The event list was narrowed by selecting substorm onsets which occurred between $\sim 0530$ MLT and $\sim 0930$ MLT, corresponding to intervals where magnetometers from the available arrays were closest to local midnight. From the identified events, five isolated substorms were chosen for detailed comparison to the Frey IMAGE-FUV onset location

Table 1. The Meyer Wavelet

\begin{tabular}{|c|c|c|c|c|c|}
\hline \multirow[b]{2}{*}{$j$} & \multicolumn{2}{|c|}{ Period (s) } & \multirow{2}{*}{$\begin{array}{l}\text { Temporal } \\
\text { Width (s) }\end{array}$} & \multirow{2}{*}{$\begin{array}{c}\text { Error } \\
\text { Estimate (s) }\end{array}$} & \multirow{2}{*}{$\begin{array}{l}\text { ULF } \\
\text { Band }\end{array}$} \\
\hline & Lower & Upper & & & \\
\hline 9 & 1.5 & 6 & 2 & \pm 1 & Pi1 \\
\hline 8 & 3 & 12 & 4 & \pm 2 & Pi1 \\
\hline 7 & 6 & 24 & 8 & \pm 4 & Pi1 \\
\hline 6 & 12 & 48 & 16 & \pm 8 & $\mathrm{Pi} 1 / 2$ \\
\hline 5 & 24 & 96 & 32 & \pm 16 & $\mathrm{Pil} / 2$ \\
\hline 4 & 48 & 192 & 64 & \pm 32 & Pi2 \\
\hline
\end{tabular}




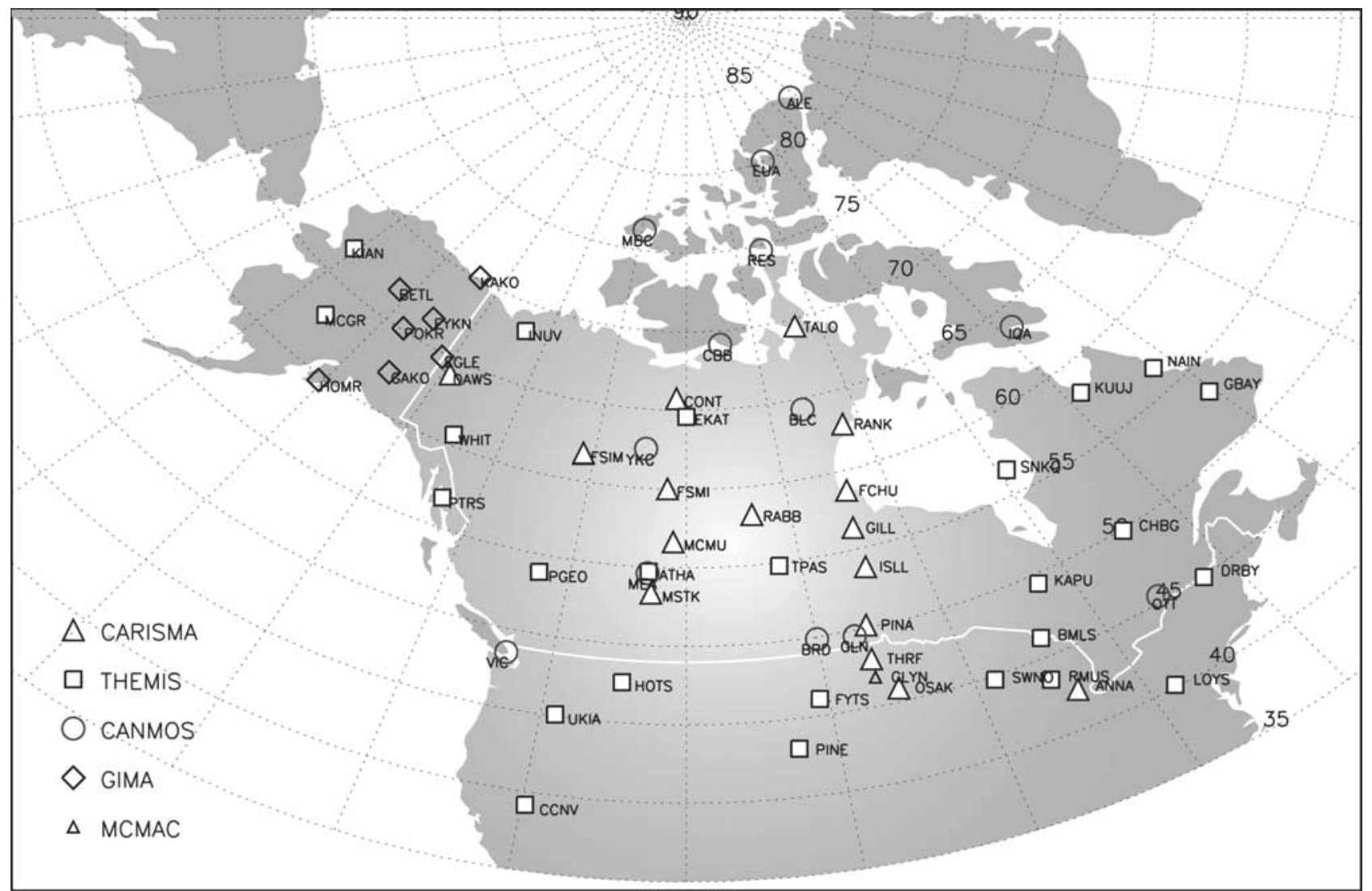

Figure 2. The location of the magnetometers comprising the Canadian Array for Realtime Investigations of Magnetic Activity (CARISMA), Time History of Events and Macroscale Interactions During Substorms (THEMIS), Canadian Magnetic Observatory System (CANMOS), Geophysical Institute Magnetometer Array (GIMA), and Midcontinent Magnetoseismic Chain (MCMAC) arrays, whose data is used in the Automated Wavelet Estimation of Substorm Onset and Magnetic Events (AWESOME) wavelet algorithm in this article.

and time. Note that due to the IMAGE orbit, all of the Frey substorms occurred where IMAGE was viewing the Southern Hemisphere and were mapped using the Tsyganenko field model to the Northern Hemisphere. Ostgaard et al. [2004, 2007] have shown that an asymmetry exists between substorm onset locations observed in the northern and Southern Hemispheres which may be described as a function of the IMF clock angle. For the events studied herein we find the IMF clock angle lies between $\sim 30^{\circ}$ and $\sim 200^{\circ}$, corresponding to a separation of less than $\sim 1.5$ MLT hours between the northern and southern hemispheric onset locations according to the Ostgaard et al. [2004, 2007] statistics. The north-south hemispheric asymmetry for each of the events studied here is roughly equal to the longitudinal separation of the magnetometers utilized in this study, thus validating the approach of using conjugate mapping of Southern Hemisphere auroral features to the Northern Hemisphere adopted here.

\subsection{Case 1: 3 June 2005}

[13] Figure 3 shows the $\mathrm{H}$ - and D-components of the magnetic field on 3 June 2005 between 0515 and 0645 UT for selected CARISMA, THEMIS and CANMOS magnetometers. A substorm is observed at $\sim 0545 \mathrm{UT}$, visible through the formation of a negative $\mathrm{H}$ bay at GILL and coincident large amplitude Pi2 oscillations. The Frey database indicates that the onset of the substorm in the Southern Hemisphere occurred in the IMAGE-FUV data at 0544:23 UT (vertical dotted line). The dashed vertical line in Figure 3 represents the initial onset of Pi1/2 activity which occurred first in the $j=5$ band (24-96 s) as determined by the AWESOME technique at the GILL magnetometer station at 0540:48 UT $( \pm 16 \mathrm{~s})$, the epicenter of ULF wave activity.

[14] The results from the AWESOME algorithm from GILL are shown in Figure 4. Figures $4 \mathrm{a}$ and $4 \mathrm{~b}$ show the $\mathrm{H}$ - and D-components of the magnetic field during the substorm interval. Figures $4 \mathrm{c}-4 \mathrm{~h}$ represent the wavelet coefficients, $\alpha_{j, k}$, for $j$ s from 9 to 4 , the horizontal gray line represents the value of the $2 \sigma$ threshold for each $j$. The substorm onset time as defined by the AWESOME technique in this case is determined from the $j=5, k=10$ wavelet coefficient; this being the first band with power that continuously exceeds the threshold. When performing the timing analysis, all wavelet bands at each station are studied. In this event the ULF wave power in the $j=5$ band is the first to rise continuously above the threshold at each station, although the time when this occurs differs from station to station. For subsequent events in this article it is sometimes the $j=6$ wavelet band which shows this behavior. We do not identify a priori band to study for any particular event, rather all wavelet bands are analyzed to identify the correct timing information. 

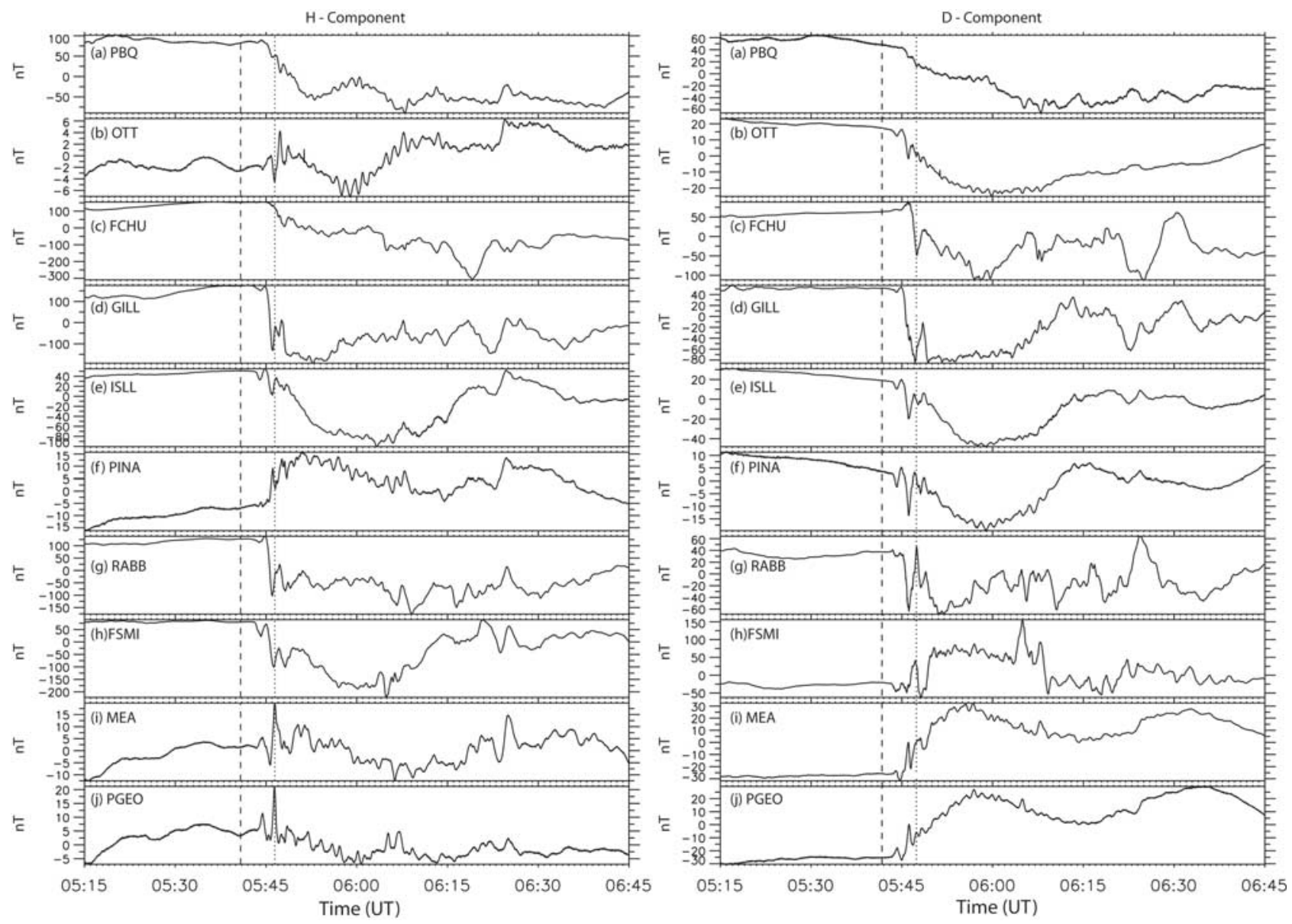

Figure 3. Selected $\mathrm{H}$ - and D-component magnetograms from 6 June 2005. The dashed line at 0540:48 UT depicts the ULF onset determined via AWESOME. The dotted line at 0546:28 UT indicates the time of Frey optical onset inferred from the Imager for Magnetopause-to-Aurora Global Exploration (IMAGE)-Far Ultraviolet Imager (FUV) instrument.

[15] Onset as determined by $\alpha_{5,10}$ is depicted by the vertical dashed line at 0540:48 UT $( \pm 16 \mathrm{~s})$. Figure 5 shows the onset of ULF activity in the $j=5$ band (Figures 5 a and $5 \mathrm{~b}$ ), and the color coded DWT spectra (Figures 5c and 5d) for GILL, the initial station, and RABB, the subsequent station to observe the onset of ULF waves. Note that we have subtracted the noise threshold from the DWT power spectra shown in Figures 5c and 5d and have normalized each wavelet band, such that power which is below the noise threshold is black and any color blocks show statistically significant power levels. Figure $5 \mathrm{a}$ is an inverse wavelet transform of the $j=5$ band shown in Figure 5b. Evident in Figures $5 \mathrm{a}$ and $5 \mathrm{~b}$ is the presence of continuous ULF power above threshold in the $j=5$ band at GILL prior to the observation of wavelet power at RABB (64 s later). Similarly, Figures 5c and 5d show a clear onset of continuous ULF wave power in the $\mathrm{j}=5$ wavelet band first at GILL, at $304 \pm 16 \mathrm{~s}$ after the start of the plot at 0535:44 UT, followed by RABB at $368 \pm 16 \mathrm{~s}$ after 0535:44 UT.

[16] Figure 6 is a minimum curvature fit of the onset times in the $j=5(24-96 \mathrm{~s})$ band, for all available magnetometer stations. The maximum error in the fitted contour times and the actual onset times is at most on the order of seconds at each station, such that the contours are an excellent representation of the onset times at each station location. Each contour is $32 \mathrm{~s}$ apart, the temporal width of each $\alpha_{5, k}$. Figure 6 shows a coherent expansion of Pi1/2 wave onset times in the $j=5$ band away from an epicenter at GILL, GILL being the first station where ULF power rises continuously above the threshold. This behavior is similar to the localization and expansion of Pil wave power onset times observed by Milling et al. [2008]. The conjugate Northern Hemisphere onset location, mapped as the negative latitude of the Southern Hemisphere onset location from the Frey database, is also shown on Figure 6 as a red cross (labeled as Frey) to the east of GILL. The blue cross (labeled T96) is the Tsyganenko 96 (T96) [Tsyganenko, 1995] magnetic field trace of the Southern Hemisphere Frey IMAGE-FUV onset location into the Northern Hemisphere. Both locations are very close to GILL, consistent with the AWESOME determined ULF onset location. It is important to note that although the onset location determined by AWESOME is limited by the spatial coverage and separation of the available magnetometer stations, the close proximity of the Frey optical and AWESOME ULF onset locations validates the effectiveness of the AWESOME algorithm.

[17] The position of the auroral electrojet and the upward and downward FACs can be estimated by comparing the magnetic bay perturbations in the H-, D-, and Z-components 


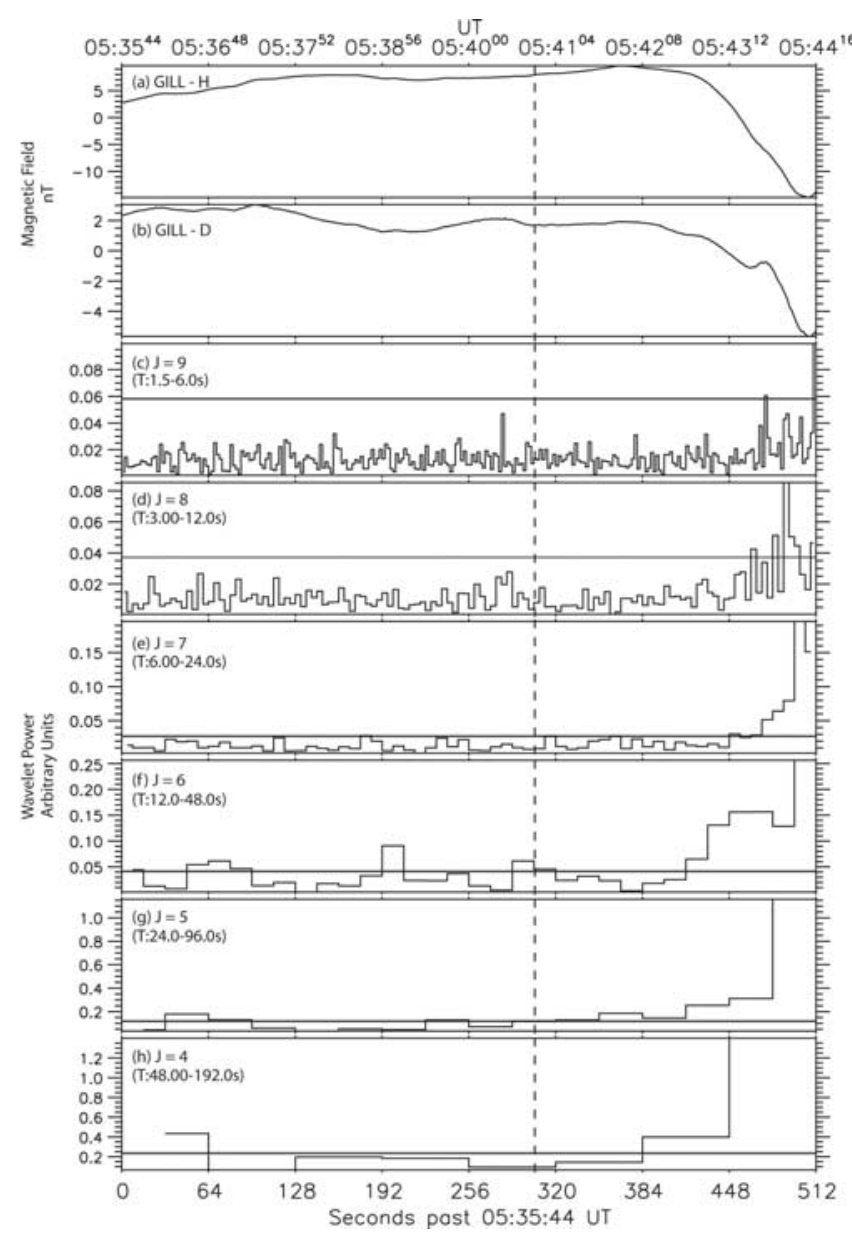

Figure 4. Implementation of the AWESOME algorithm at the GILL magnetometer. $(\mathrm{a}, \mathrm{b}) \mathrm{H}$ - and D-components of the magnetic field. $(\mathrm{c}-\mathrm{h}) \mathrm{ULF}$ wave power in the wavelet coefficients $j=9-4$, respectively. The horizontal gray line in Figures $4 \mathrm{c}-4 \mathrm{~h}$ represents the threshold for each $j$ band. The dashed line depicts the onset at 0540:48 UT represented by the $j=5, k=10$ wavelet coefficient in Figure $4 \mathrm{~g}$.

to a SCW model consisting of dipolar FACs connecting through an ionospheric closure current [e.g., Cramoysan et al., 1995]. These authors have determined the amplitude and the sign of the initial magnetic perturbations as a function of latitude and longitude with respect to a model SCW [see also Smith et al., 2002, Figure 8]. By fitting the substorm bays observed on this day it is estimated that the central meridian of the SCW lies between MCMU and PINA; however due to limited coverage, an accurate determination of the east-west extent and latitude of the electrojet was difficult. We do note that the AWESOME determined onset location is east of the central SCW meridian, in the direction toward the downward FAC element.

\subsection{Cases 2 and 3: 17 and 20 July 2005}

[18] Three substorms on 17 and 20 July 2005 are analyzed in this section providing further validation for the AWESOME technique. Figures 7 and 8 show the H-component of the magnetic field from selected stations for 17 and 20 July 2005, respectively. Two magnetic substorms were identified in the Frey substorm database on 17 July 2005: one at 0714:15 UT (first dotted line in Figure 7) and the second at 0848:11 UT (second dotted line in Figure 7). A third isolated magnetic event on 17 July 2005 can be seen in the highlighted section of Figure 7 between 0800 and 0830 UT, and will be addressed in more detail in the next subsection. The first dashed line depicts the onset time at FSMI which occurred first in the $j=6$ frequency band as determined by the wavelet algorithm at 0706:28 UT ( $\pm 8 \mathrm{~s})$. Note however that the IMAGE determination of substorm onset during this event is relatively uncertain, $\sim 9 \mathrm{~min}$, as IMAGE was experiencing tracking difficulties; between $\sim 0705$ and $\sim 0714$ the onset region shifted out of the field of view of IMAGE and the onset time may in actuality be more consistent with the ULF determined onset time. The second dashed line depicts the ULF onset in the $j=5$ wavelet band for the final event, which occurs first in this band at GAKO, at 0835:48 UT $( \pm 16 \mathrm{~s})$. The Frey substorm database identifies a single substorm on 20 July 2005 at 0530:53 UT, identified by the dotted line of Figure 8, the dashed line showing the DWT ULF onset which occurred first in $j=5$ at 0524:24 UT ( $\pm 16 \mathrm{~s})$ at MCMU.

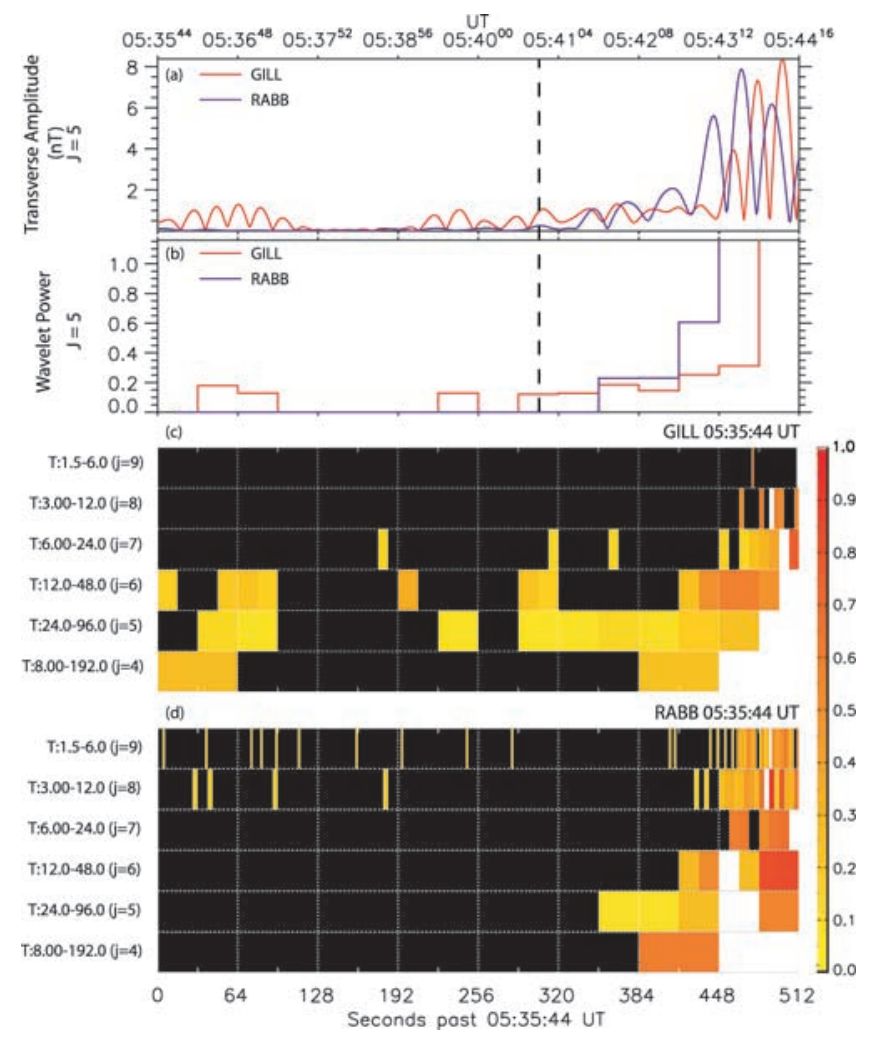

Figure 5. (a, b) Onset of ULF waves in the $j=5$ band for the GILL (red) and RABB (blue) magnetometers. Figure 5a shows the inverse transform of the transverse ULF amplitude determined from the $j=5$ wavelet coefficients shown in Figure $5 \mathrm{~b}$. The dashed line depicts the ULF onset at the GILL magnetometer station at 0540:48 UT, $64 \mathrm{~s}$ before the onset at RABB. (c, d) Normalized Pi1 and Pi2 wavelet power spectra for $j \mathrm{~s} 9-4$ at GILL and RABB, respectively. The $x$ axis denotes time, and the $y$ axis denotes period. Black represents wavelet coefficients below the determined threshold for each $j$; yellow-orange-red-white are coefficients rising above the threshold in increasing amplitude. 


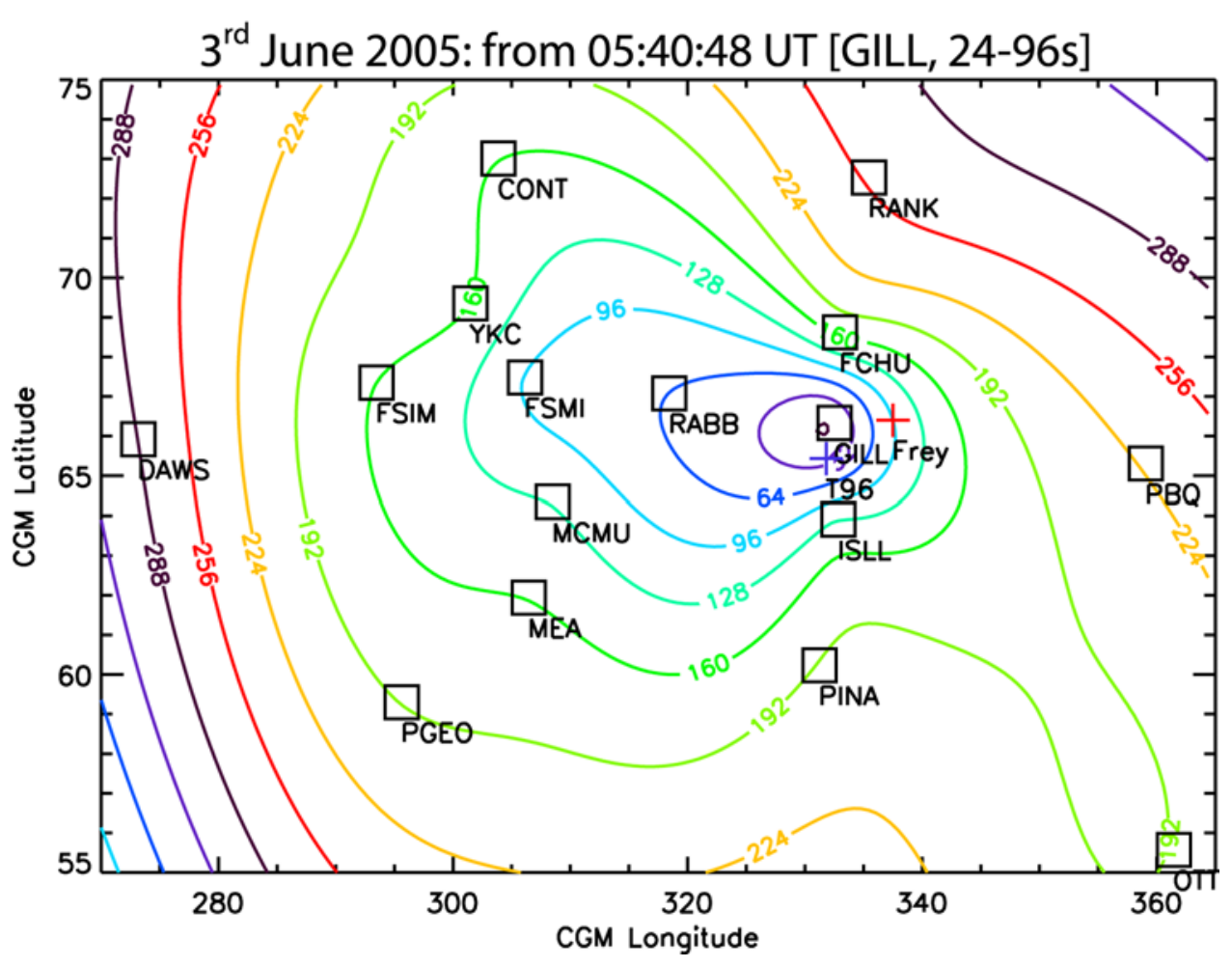

Figure 6. Contour plot of $j=5$ onset times determined by AWESOME on 6 June 2005. ULF onset is observed first at the GILL and expands coherently in both latitude and longitude. The red cross denotes the conjugate Northern Hemisphere onset location in the Frey substorm database. The blue cross is the Tsyganenko field trace of the Southern Hemisphere onset location to the Northern Hemisphere.

[19] Figure 9 shows a minimum curvature contour plot of the delay in the onset of ULF wave activity (same format as Figure 6) for these three events on 17 and 20 July 2005. Figure 9a shows the expansion of the $j=6$ onset for the first substorm and Figure $9 \mathrm{~b}$ the expansion of the $j=5$ onset of the second substorm on 17 July 2005 . Figure $9 \mathrm{c}$ shows the expansion of ULF wave $(j=5)$ onset times during the substorm observed on 20 July 2005. The location of substorm onset mapped to the Northern Hemisphere is labeled by the red and blue crosses. The red cross is the conjugate Northern Hemisphere onset location as determined by Frey et al. [2004] (negative latitude of the Southern Hemisphere onset location) and the blue cross is the T96 magnetic trace of the Frey onset location in the Southern Hemisphere to the Northern Hemisphere. Apparent during each substorm is a clear propagation of the onset ULF wave activity away from a localized epicenter. This epicenter occurs at FSMI, GAKO, and DAWS in Figures 9a, 9b, and 9c, respectively, in each case lying close to the onset location as determined by Frey et al. [2004] mapped into the Northern Hemisphere.

[20] The Cramoysan et al. [1995] SCW model analysis of the H-, D-, and Z-component bays for these three substorms yields an estimate for the latitude and meridians of the electrojet and upward and downward FACs. The electrojet for the first substorm observed on 17 July 2005 (0706:28 UT) was centered approximately between MCMU and RABB in longitude, and between MEA and FSMI in latitude. The downward FAC system lies east of the "Churchill" line of magnetometers (FCHU-GILL-ISLL-PINA) and the upward FAC approximately in the VIC meridian. For the second substorm on 17 July 2005 (0835:48 UT) the central meridian of the auroral electrojet was east of DAWS, and between GAKO and HOME in latitude. Owing to limited coverage to the east and west for this substorm accurate determination of the meridians of the FAC elements was not possible. On 20 July 2005 the magnetic bays are small and combined with limited midlatitude coverage, we were unable to accurately determine the location of the electrojet and corresponding meridians of the FAC.

\subsection{Case 4: Isolated Substorm Between 0800 and 0830 UT, 17 July 2005}

[21] The bays associated with the second event observed on 17 July 2005 (shaded region in Figure 7) are small and localized. The highlighted section between 0800 and 0830 UT in Figure 7 is shown in detail in Figure 10. Evident in Figures 10a and 10b is the formation of a small amplitude negative bay in the H-component at RABB and FSMI at $\sim 0810$ UT. The remaining stations show limited deflection of the H-component magnetic field; however Pi2s are readily observed which suggests the formation of a localized substorm or perhaps a pseudobreakup [e.g., Baker et al., 1996, and references therein; Voronkov et al., 2003].

[22] Though the extent of this magnetic event is limited, the onset and subsequent expansion of ULF waves can still be characterized. Figure 11 shows a contour plot of the onset and expansion of ULF waves in the $j=5$ (24-96 s) wavelet band (same format as Figure 6). The ULF wave onset is observed simultaneously at the FSIM, FYKN and DAWS magnetometers at 0807:00 UT $( \pm 16$ s, black line 


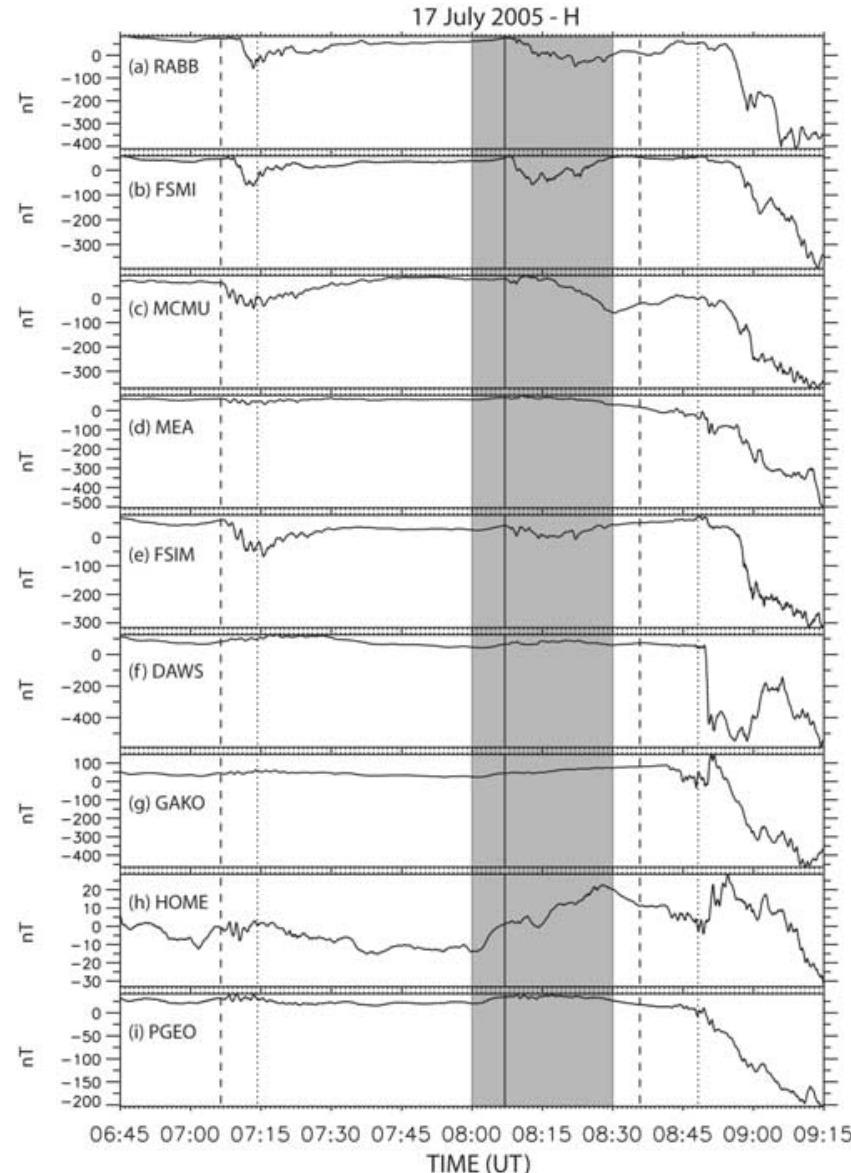

Figure 7. The H-component magnetic field from select magnetometers on 17 July 2005. The dashed lines, 0706:28 UT and 0835:48 UT, represent the ULF onset. Dotted lines, 0714:15 UT and 0848:11 UT, depict the optical onset times. The highlighted region is a localized pseudobreakup, discussed in section 3.3 and Figure 10.

Figure 10) suggesting that the onset of ULF power lies between these three stations. Similar to the previous four events, the ULF wave onset expands coherently across the available magnetometer stations.

[23] A localized auroral brightening is seen by the IMAGE-FUV instrument at approximately 0808 UT on 17 July 2005 (data not shown), coincident in time (to within instrumental resolution) with the onset of ULF waves at the FSIM, FYKN and DAWS magnetometer stations. Unfortunately during this interval, the IMAGE satellite experienced tracking difficulties which caused the auroral oval to shift in and out of the field-of-view of the FUV instrument. As such, the limited spatial and temporal expansion of the auroral oval observed by IMAGE-FUV fails to meet the criteria set forth by Frey et al. [2004] to be included in the substorm database; however the auroral brightening seen in the FUV data suggests that the intensification may be indicative of a pseudobreakup or other intensification which is not followed by auroral breakup (i.e., the poleward expansion and breakup of the main arc into multiple arcs). This localized event suggests that the AWESOME technique may also be utilized to determine the onset of ULF phenomena and their resulting spatial and temporal expansion during pseudobreakup events [see Rae et al., 2008].

\subsection{Compound Substorm Expansion Event on 18 November 2005}

[24] While the wavelet algorithm is able to successfully characterize the ionospheric arrival times of Pil ULF waves during isolated substorms onsets, it is clearly more difficult to determine the onset timing and propagation during events in which multiple onsets, localized pseudobreakups, or ULF precursors associated with discrete arc brightenings are observed prior to the full expansion phase onset. One such compound event was observed on 18 November 2005. Figure 12 shows the AWESOME determined timings of the first ULF power to exceed threshold, which occurs in this instance in the $j=5$ band. The initial disturbance is observed first at the FCHU and GILL magnetometers at 0605:24 UT $( \pm 16 \mathrm{~s})$ followed by RABB and YKC $64 \mathrm{~s}$ later. Apparent in Figure 12 are two regions from which the $j=5$ waves expand; one centered on the FCHU-GILL magnetometers and the second centered about the YKC magnetometer. The Frey database indicates that substorm onset occurred at 0609:29 UT, in the Southern Hemisphere at the CGM latitude and longitude of $-65.93^{\circ}, 301.66^{\circ}$ respectively. Figure 12 shows the inverse magnetic location as a red cross

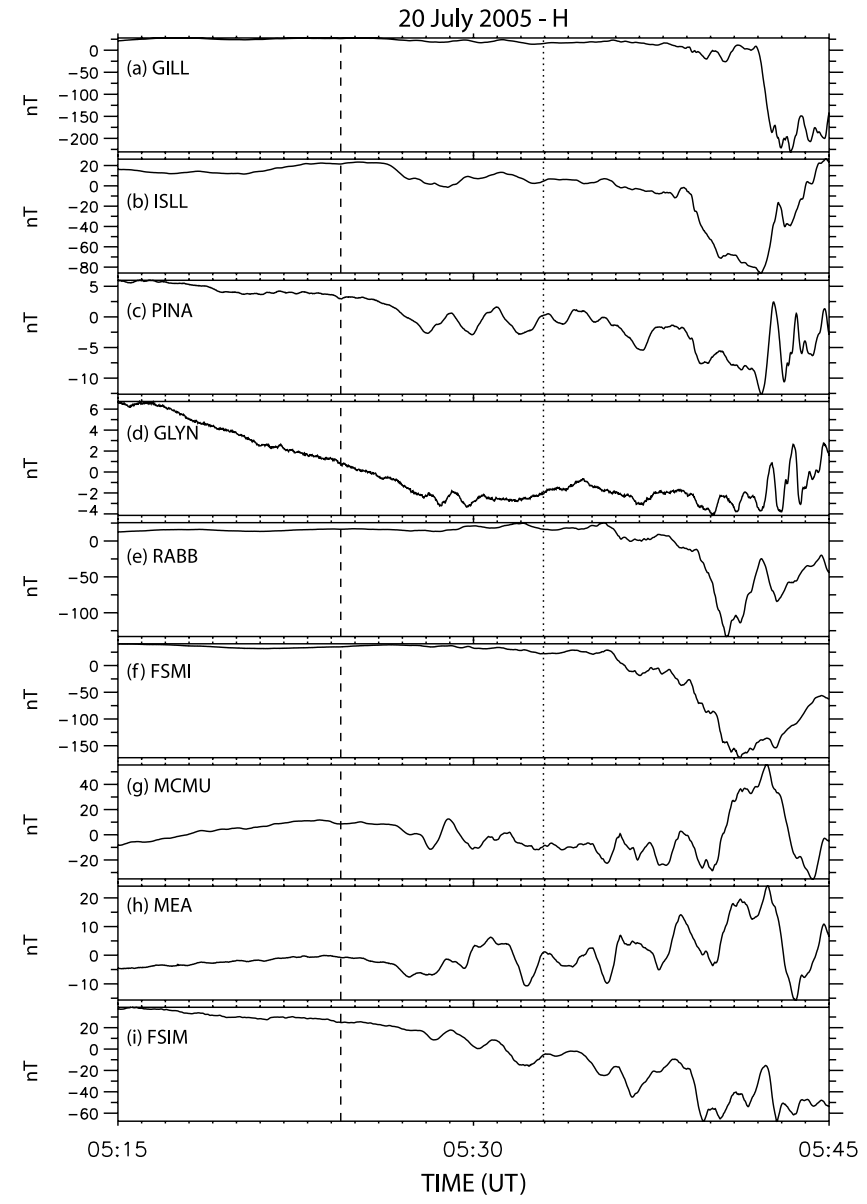

Figure 8. Select H-component magnetic time series on 20 July 2005. The dashed line is the ULF onset at 0524:24 UT, and the dotted line is the optical onset at 0532:57 UT. 
(a) $17^{\text {th }}$ July 2005 : from 07:06:28 UT [FSMI, 12-48s]

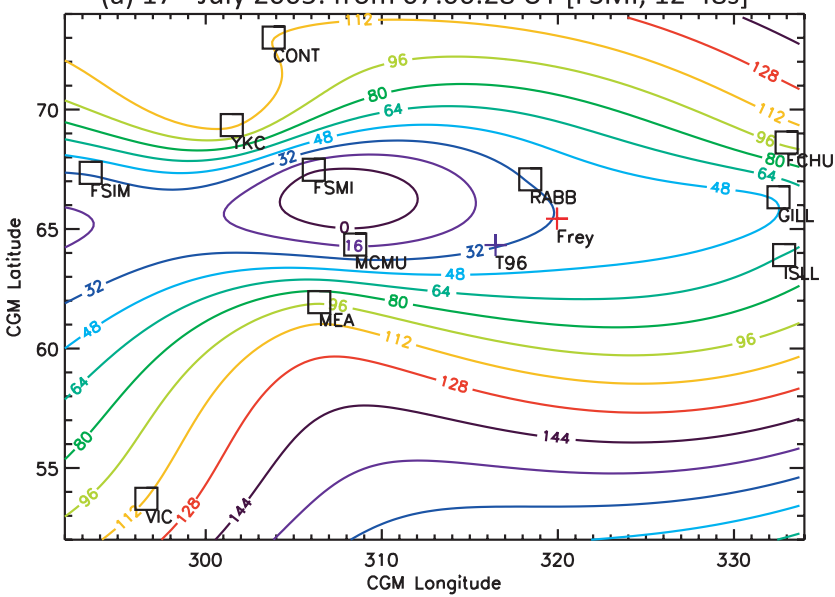

(b) $17^{\text {th }}$ July 2005 : from 08:35:48 UT [GAKO, 24-96s]

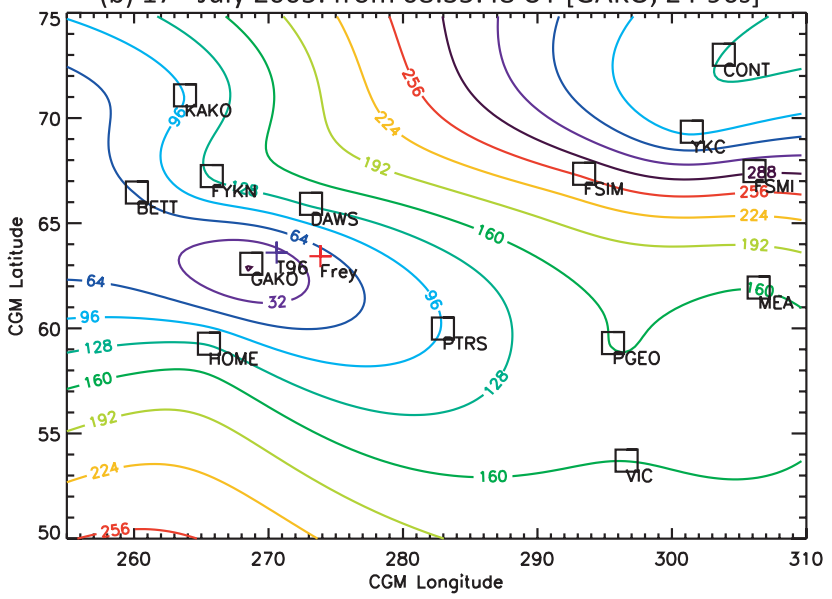

(c) $20^{\text {th }}$ July 2005: from 05:24:24 UT [MCMU, 24-96s]

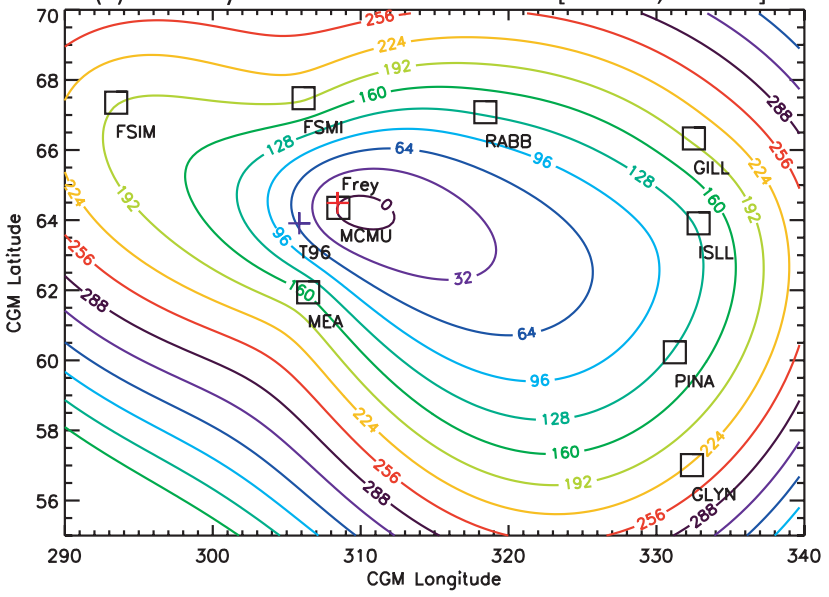

Figure 9. Contours of the ULF onset time for (a) the initial substorm on 17 July 2005 in the $j=6$ wavelet band; (b) the second substorm on 17 July 2005 in the $j=5$ wavelet band; and (c) the 20 July 2005 substorm in the $j=5$ wavelet band. The red crosses are the conjugate Northern Hemisphere Frey onset locations. Blue crosses are the T96 field trace of the Southern Hemisphere optical onset location to the Northern Hemisphere. at $+65.93^{\circ}$. The T96 trace to the Northern Hemisphere is located at CGM latitude and longitude of $65.71^{\circ}$ and $303.86^{\circ}$, shown in Figure 12 as a blue cross. The conjugate Frey and T96 Northern Hemisphere onset locations both lie between the MCMU, FSMI and FSIM magnetometers. As described above the wavelet algorithm indicates the ionospheric onset occurred first in the $j=5(\mathrm{Pi} 1 / 2)$ wavelet band at the FCHU and GILL magnetometers. However, the second YKC epicenter of the Pi1/2 activity is approximately coincident with the two mapped Frey onset locations. Such an expansion pattern suggests the Pi1/2s observed at FCHU and GILL might be a localized magnetic phenomenon which occurs prior to the full onset of the substorm.

[25] Rae et al. [2008] have shown that prior to the full expansion of the aurora during substorm breakups, spatially localized auroral brightening can be observed coincident with Pi1 and Pi2 wave packets. From analysis of these other events, we suggest that on 18 November 2005, similar localized auroral signatures near the GILL and FCHU magnetometers are responsible for the initial ULF activity observed prior to the full onset of the substorm which begins $64 \mathrm{~s}$ later and has an epicenter to the west of these stations. Indeed the GILL meridian scanning photometer (MSP) in the Northern Solar Terrestrial Array (NORSTAR) array of

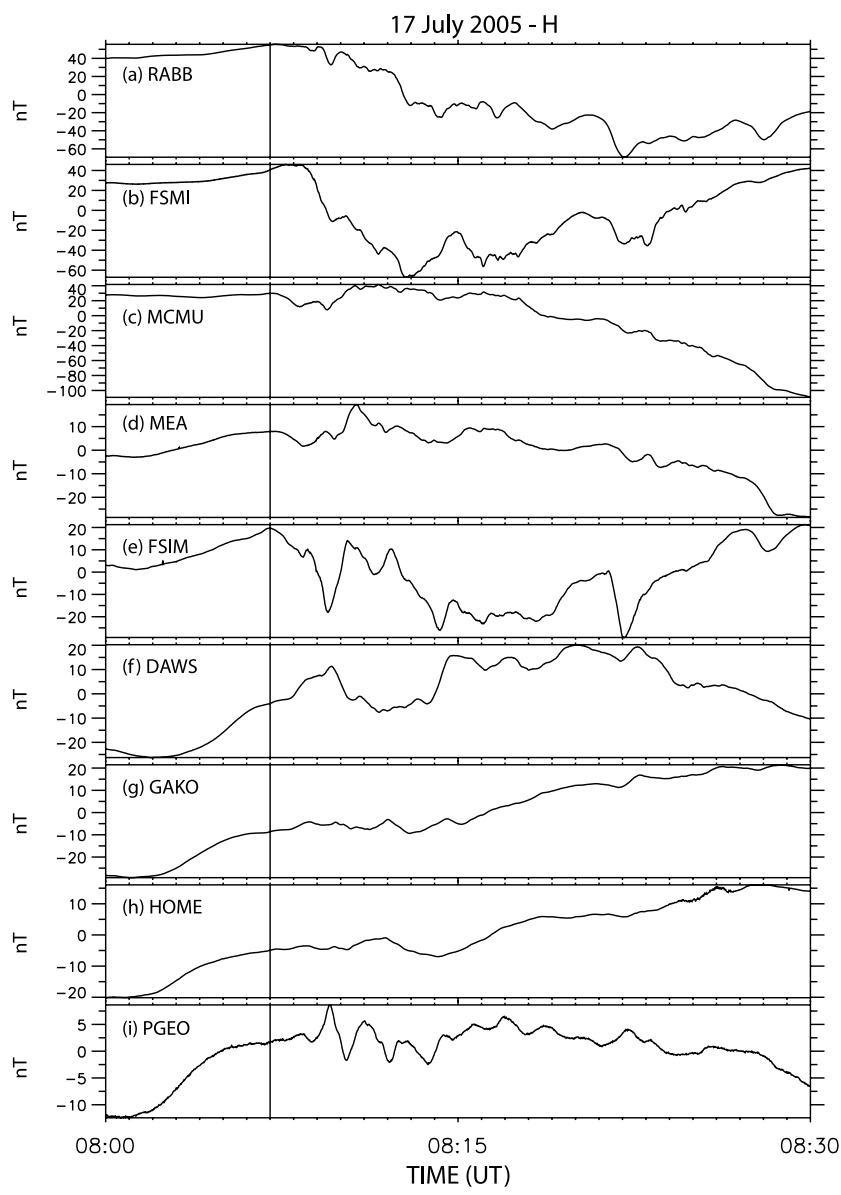

Figure 10. An expanded view of the highlighted portion of Figure 7. The black line depicts the ULF onset of a pseudobreakup event at 0807:00 UT; no optical onset for this event is identified in the Frey substorm database. 


\section{$17^{\text {th }}$ July 2005: from 08:07:00 UT [FYKN/DAWS/FSIM, 24-96s]}

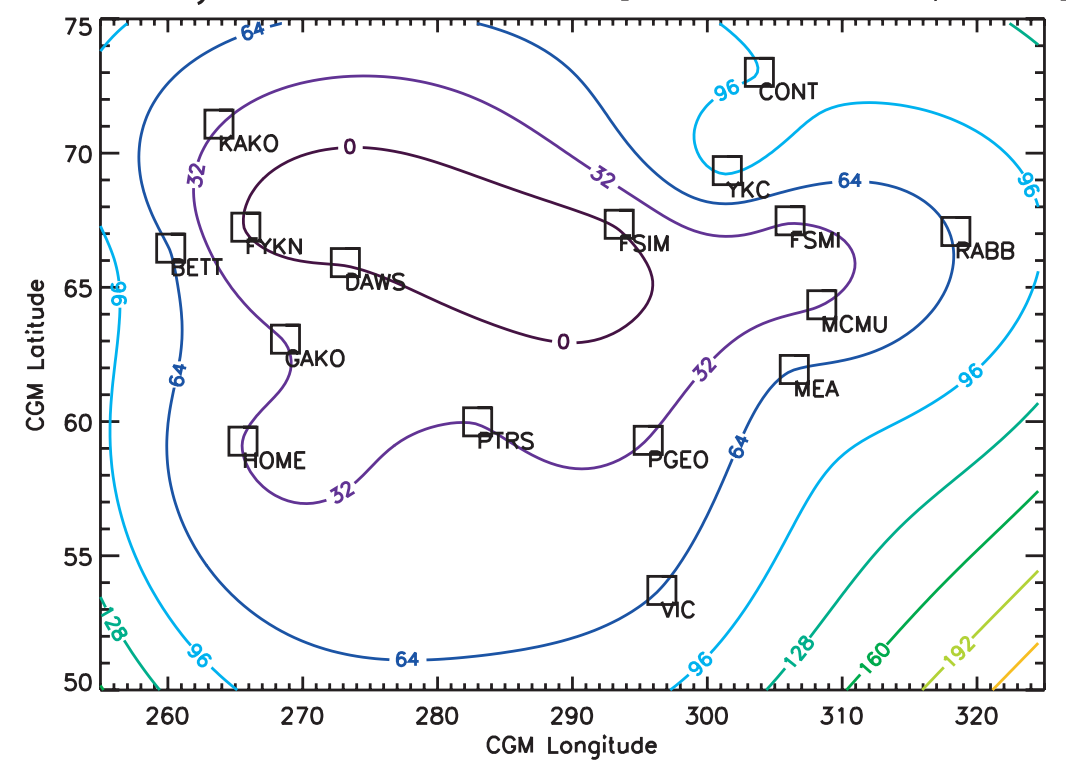

Figure 11. A contour plot of the ULF onset times for the pseudobreakup observed between 0800 and 0830 UT on 17 July 2005. The first ULF onset occurred in the $j=5$ wavelet band.

the Canadian Space Agency Canadian Geo-Space Monitoring program (data not shown) shows evidence of local auroral brightening at approximately 0605 UT several minutes prior to the substorm breakup.

[26] Indeed, it is possible to interpret the disturbance observed at FCHU and GILL as a precursor and identify a second intensification of $\mathrm{Pi} / 2$ activity after the initial rise of Pi1/2 ULF activity above presubstorm background noise. This produces a more coherent pattern of temporal expansion of Pil/2 signals away from a single epicenter close to YKC, near the Frey onset location. Figure 13 shows results which arise when the time of the second burst of Pi1/2 activity is considered at FCHU and GILL. Note there is now a clearer expansion of substorm onset related Pi1/2 activity, centered on a single epicenter.

[27] Figure 14a shows the H-component magnetic field observed at the YKC (gray) and FCHU (black) magnetometers. The dashed gray lines depict the initial onset of ULF power at FCHU and the second intensification of ULF power, the black dashed line indicates the onset ULF power at YKC and the dotted line depicts the onset time identified in the Frey substorm database. Figures $14 \mathrm{~b}$ and $14 \mathrm{c}$ show the inverse wavelet transform of the $j=5$ band the $j=5$ wavelet power, respectively. Apparent in Figure 14c are two packets of wave power observed at FCHU (gray), indicated by the gray dashed lines, and a continuous rise in power at YKC (black), identified as the onset of the substorm (dashed black line). The initial packet observed by FCHU is associated with the localized auroral brightening apparent in the GILL MSP and the second packet is the onset of the substorm identified by IMAGE in the Frey database. While the wavelet algorithm is an excellent tool for determining substorm onset and the resulting expansion of ULF waves, this example illustrates that care must still be taken when identifying Pils and Pi2s and associating them with substorm onset, as arc brightenings and auroral dynamics can also be associated with Pi1/2 activity which will also be detected by the AWESOME DWT algorithm.

[28] The position and extent of the SCW can also be estimated by the initial H, D and $\mathrm{Z}$ bays seen following the onset of this substorm. The approximate center of the SCW lies at a longitude between FSMI and RABB and at a similar latitude to both stations. The upward FAC meridian lies between FSIM and DAWS and the meridian of the downward FAC system lies to the west of the Churchill line between RABB and GILL. In this case the optical and Pi1/2 ULF onset locations lies between the upward and downward FAC element.

\section{Discussion}

[29] Each of the five substorms from the Frey database, as well as the localized pseudobreakup observed at $\sim 0800$ UT on 17 July 2005, studied here show a universal picture of a coherent expansion of the onset of Pi1/2 ULF waves away from a localized onset epicenter in the ionosphere. Angelopoulos et al. [2008] showed that the westward traveling surge (WTS) expands azimuthally in the ionosphere at a rate of $\sim 15^{\circ}$ in longitude/min. The typical longitudinal expansion rate of the onset of $\mathrm{Pi} 1 / 2$ ULF waves in the events studied here is $\sim 30 \mathrm{~s}$ per $10^{\circ}$ of magnetic longitude. The observed Pi1/2 ULF wave onset expansion rate reported here is hence faster than that of the WTS. This is in agreement with the observations of Samson and Harrold [1985], who concluded that the excitation mechanism of ULF waves at expansion phase onset is different than that responsible for the auroral break up and subsequent propagation of the WTS.

[30] Using a fit to the substorm magnetic bays [Cramoysan et al., 1995], the location of the SCW in four of the six events was compared to the AWESOME-determined Pil onset location. In these four events the center of the latitude of the electrojet in the SCW was found to be co-located with the 


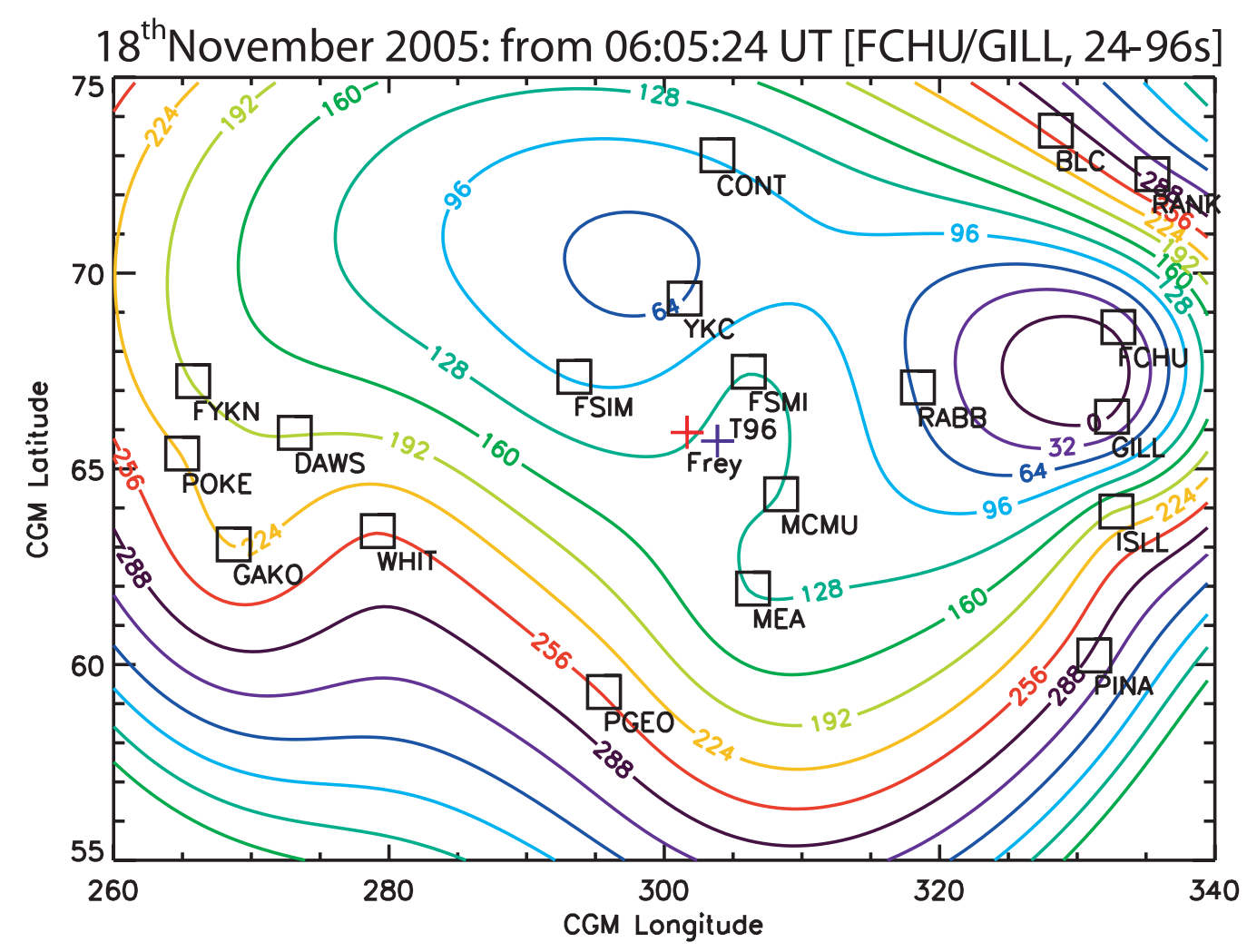

Figure 12. Contour plot of ULF onset times in the $j=5$ wavelet band on 18 November 2005, during a compound magnetic substorm. Conjugate optical onsets are indicated by the red (Frey) and blue (T96 field trace) crosses.

latitude of both the (conjugate) optical Frey IMAGE-FUV and AWESOME Pi1 ULF wave onset locations. Of these four events, two were located in a region of the North American sector in which there was sufficiently dense midlatitude and auroral magnetometer coverage surrounding the onset location to estimate the meridians of the FAC elements in the SCW. Milling et al. [2008] have suggested that the location of the epicenter of Pil ULF wave onset is co-located with the region in which the downward FAC element subsequently develops. On 17 July 2005 the onset location was found to lie between the upward and downward FAC elements. On 18 November 2005 both the Pi1/2 ULF and Frey optical onsets were close to the upward FAC element of the SCW, though as described in section 3.4 the observed substorm was compound in nature. This evidence suggests that a localized auroral brightening occurred close to the FCHU-GILL magnetometers prior to the full onset of the substorm which occurred $64 \mathrm{~s}$ later to the west and centered close to YKC. For this event, the ULF epicenter and the Frey optical onset appear to be between the locations of the upward and downward FAC meridians. Note however that the spatial resolution available to the SCW technique is limited by the separation of the stations in the array such that spatial errors in determining the meridians of the SCW elements can be as large as the longitudinal station separation. Note also, as shown by Cramoysan et al. [1995; see also Gelpi et al., 1987; Lester et al., 1989; Smith et al., 2002], care must be taken in inferring the meridian and latitude of the SCW system since there is flaring of the zero crossings in the average magnetic bay perturbations, and thus the longitudinal magnetic bay structure is a function of latitude.

[31] The DWT analysis presented in this article demonstrates a clear relationship between the location of the first ionospheric Pil/2 activity, and the location of the globalscale substorm auroral brightening which can be seen with the global-scale FUV imager on the IMAGE satellite validated via comparison to five substorms in the Frey substorm list. Further, independent location of the SCW current system by analysis of the substorm bays shows a close correspondence between the latitude of the electrojet and the Pi1/2 onset, and the meridians of the center of the SCW or the meridian of the downward FAC element, which subsequently develops. In their recent article, Milling et al. [2008] also found a spatial correspondence between the location of the ULF onset and the location where the downward FAC element subsequently develops.

[32] One obvious interpretation for the close spatial correspondence between the SCW central meridian/downward FAC element and the Pi1/2 onset epicenter is that the Pi1/2 epicenter reflects the field-aligned ionospheric image of the perturbations created in the magnetosphere by the mechanism triggering expansion phase onset. It is possible that the Pil $/ 2(j=5$ or 6$)$ waves are a signature of the first seconds of the substorm expansion phase onset process in the ionosphere. Significantly, the rate at which the Pi1/2 onset propagates in latitude and longitude in the ionosphere is much slower than the time take for shorter-period (1- to 10-s period) PilB signals to propagate in the Earth-ionosphere 


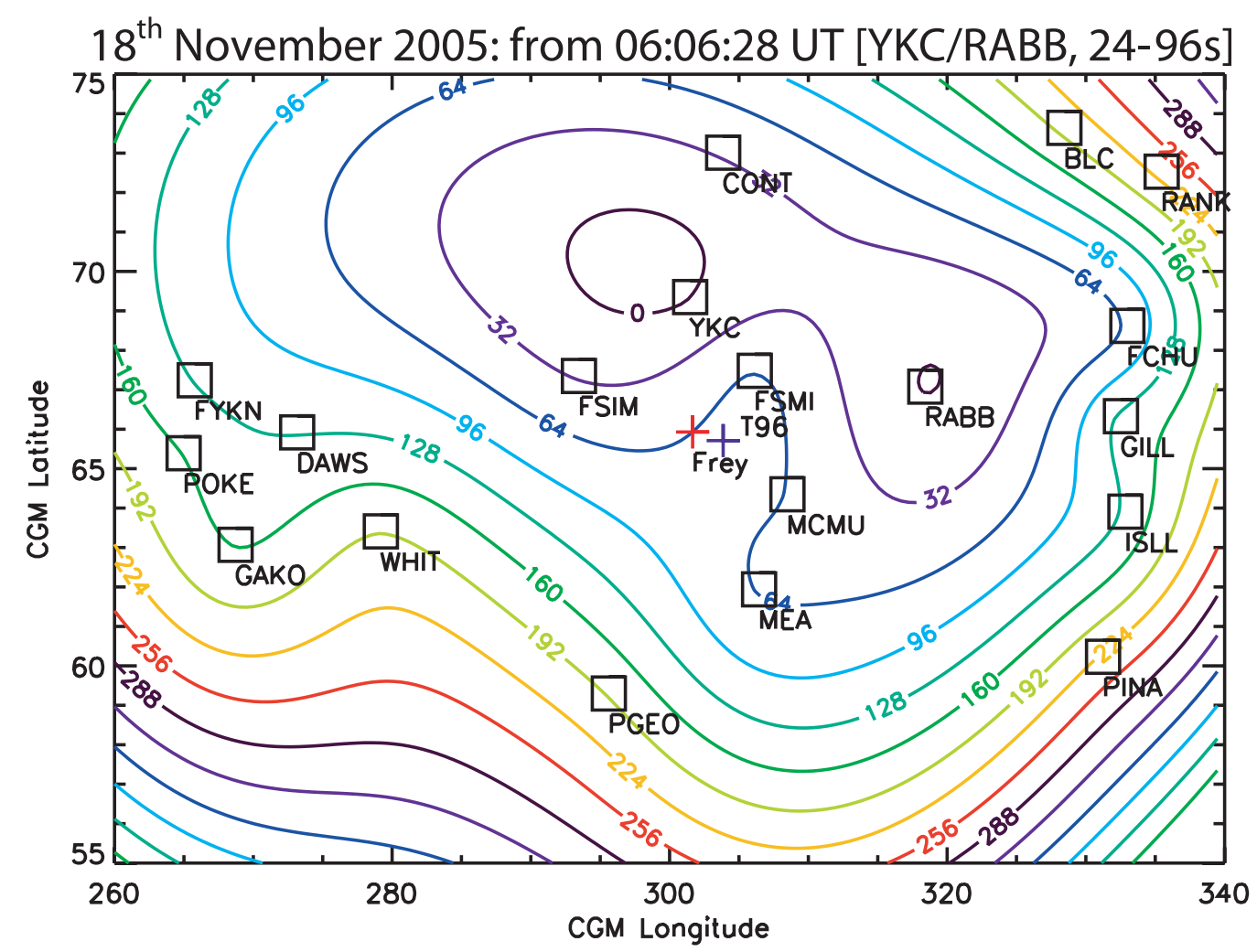

Figure 13. Contour plot of the ULF intensifications in the $j=5$ wavelet band on 18 November 2005, during a compound magnetic substorm. Conjugate optical onsets are indicated by the red (Frey) and blue (T96) crosses.

waveguide [Lysak, 1988], ruling out a role for the waveguide in the expansion of the Pil onset signal reported here. Similarly, assuming an Alfvén speed of $1400 \mathrm{~km} / \mathrm{s}$ in the magnetosphere at geosynchronous orbit $(B=100 \mathrm{nT}, n=$ $3 / \mathrm{cc}$ ) results in an azimuthal propagation speed in the ionosphere of $\sim 10 \mathrm{~s}$ to travel $1 \mathrm{~h}$ of MLT of longitude. This Alfvénic propagation time is thus much faster than the expansion of the Pi1/2 onset times observed by the individual magnetometers in the ionosphere, suggesting that any cross-field propagation of a source region of the onsetrelated Pi1/2s in the magnetosphere occurs much slower than the local Alfvén speed. It is possible that the Pi1/2 signals identified by the wavelet transform represent the arrival of an Alfvén wave excited by disturbances which may map to a localized region of the magnetotail where the cross-tail current is diverted into the ionosphere to form the SCW. In principle such a signal could be generated in a localized region by $\mathrm{CD}$ in the magnetotail, or in the process of flow braking [e.g., Shiokawa et al., 1997, 1998] following reconnection further tailward in the NENL model. If however the region of the nightside magnetosphere is extremely elongated then the initial expansion of the Pi1/2 may be a result of the direct propagation of an Alfvén wave along the plasma sheet boundary layer from the reconnection region corresponding to the NENL. Conjugate studies of future events combining in situ data from the magnetotail with ionospheric onset diagnosis using AWESOME will enable this hypothesis to be tested and perhaps enable the driver of Pi1/2 onset waves to be uniquely determined.
[33] Despite the need for further in situ studies, our observations are consistent with the following scenario. The first Pil/2 waves characterize the arrival in the ionosphere of Alfvénic disturbances triggered by the expansion phase onset process in the magnetotail. Subsequently, the power in the Pi1 and Pi2 bands gradually increases for $\sim 2$ min. During this time the onset arc intensifies, and after $\sim 2-3$ min auroral breakup follows, characterized by a rapid and large increase in Pil and Pi2 power. The initial lowpower Pi1/2s reported here can therefore be used to time and locate the very first signatures of onset in the ionosphere. Auroral breakup and the build up of the FAC in the SCW are only established later as a result of the propagation and reflection of large amplitude Alfvén waves, historically characterized in the magnetograms as Pi2s. Finally, the westward drift of substorm injected ions in the magnetosphere creates a westward expansion of the spatially distributed upward FAC in the WTS. This expansion of the WTS most likely occurs as the result of $\mathrm{j}_{\|} \propto \nabla \mathrm{P} \times \nabla \mathrm{V}$, where $\mathrm{P}$ is the pressure and $\mathrm{V}$ is the flux tube volume [e.g., Vasyliunas, 1970], driving an upward FAC which expands westward under the pressure gradient front arising from gradient curvature drift of the injected ions [cf. Lyons, 1995]. The large-scale auroral brightening identified in the FUV images characterizes the onset of the substorm in the Frey database several minutes after the time that ULF wave power first exceed the quiet time threshold.

[34] A very important question concerns the origin of the Pi1/2 waves seen in the ionosphere, especially the physical implications of the existence of the Pil/2 wave onset 


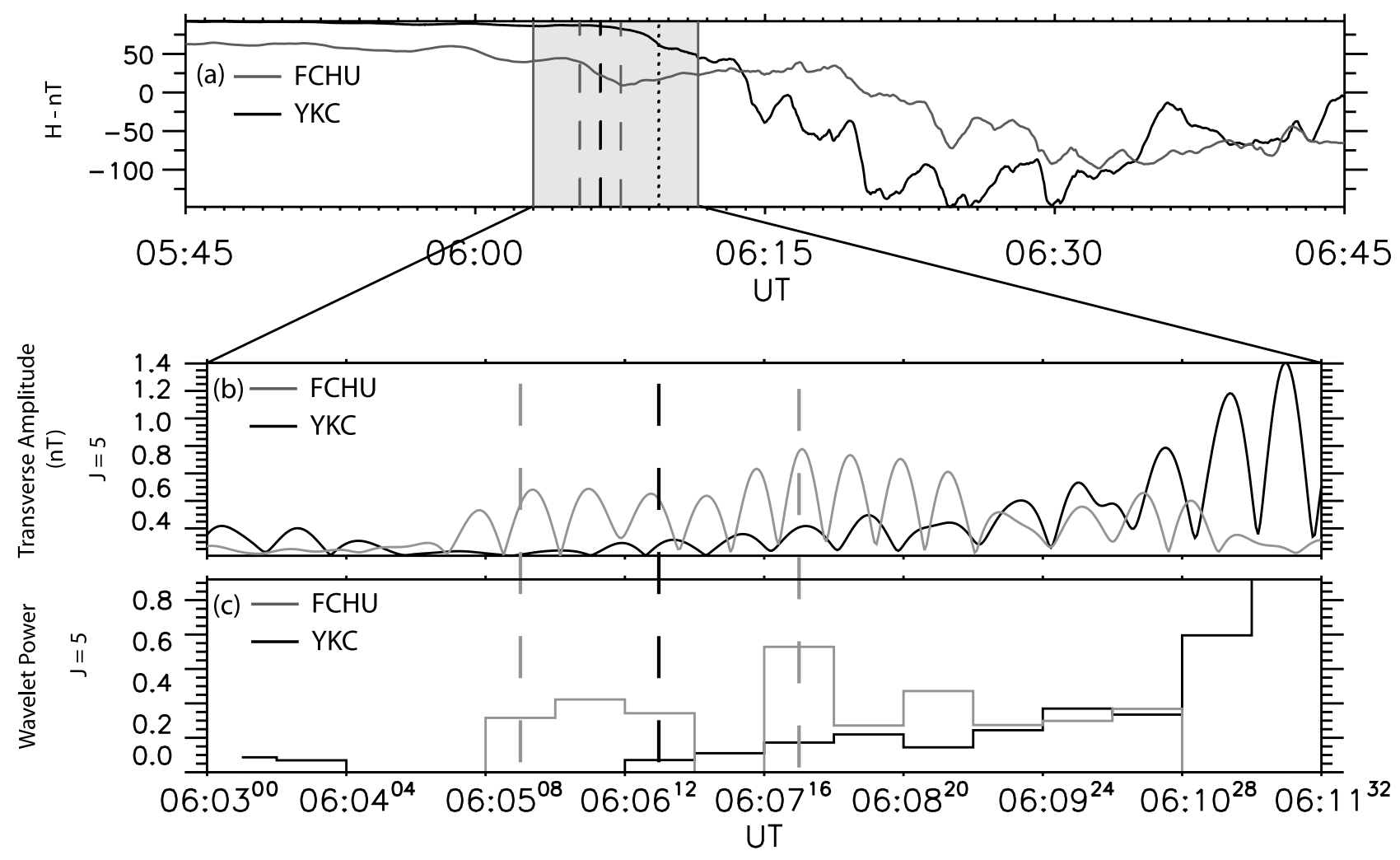

Figure 14. (a) H-component magnetic field observed by the YKC (black) and FHCU (gray) magnetometers during the compound substorm on 18 November 2005. The first gray dashed line indicates the onset of ULF wave power at FCHU associated with a localized brightening of the aurora; the second gray dashed lined depicts intensification of $\mathrm{Pi} / 2$ power; the black dashed line indicates the onset of ULF power observed by YKC and the onset of the magnetic substorm identified in the Frey substorm database. (b) Inverse wavelet transform of the $j=5$ wavelet band for the YKC (black) and FCHU (gray) magnetometers. Figure $14 \mathrm{~b}$ is the $j=5$ wavelet power at YKC (black) and FCHU (gray) magnetometers. The dashed lines in Figures 14a and 14b are the same as those in Figure 14a.

epicenter in the ionosphere. Previous optical studies have observed the occurrence of small-scale auroral undulations immediately prior to expansion phase onset [e.g., Donovan et al., 2006; Liang et al., 2008; Rae et al., 2008; I. J. Rae et al., Near-Earth initiation of terrestrial substorm onset, submitted to Journal of Geophysical Research, 2008]. In order to link the optical and magnetic manifestations of expansion phase onset, Rae et al. (submitted manuscript, 2008) determined that the epicenter of long-period Pil ULF wave activity in the ionosphere is coincident in both time and space with these small-scale auroral beads or arc undulations that form on the most equatorward arc prior to auroral breakup. Furthermore, Rae et al. (submitted manuscript, 2008) determined that the most poleward arcs remained undisturbed during the development of this Pi1/2 signature and during the development of the auroral beads. This suggests that the Pi1/2 onset is an ionospheric signature of a CD mechanism in the near-Earth plasma sheet, the signatures evolving for several minutes prior to the disruption and breakup of more poleward discrete arcs which map to the nightside central plasma sheet. Alternatively, in an extremely stretched tail the initial expansion of Pil/2 waves in the ionosphere may extend into the distant magnetotail, the onset of ULF wave activity thus being associated with the triggering of reconnection and the substorm expansion phase, consistent with the NENL model. However, in such a scenario the development of auroral undulations at latitudes lower than the preexisting and quiescent poleward arc must be successfully explained (e.g., Rae et al., submitted manuscript, 2008). More studies including in situ data are needed to provide conclusive evidence of the location and driver of the initial magnetospheric disturbance.

\section{Conclusions}

[35] In this article we describe the implementation and validation of a discrete wavelet transform algorithm, AWESOME, which enables the determination of the magnetic onset of Pi1/2 ULF waves in the ionosphere during magnetic substorms. The wavelet algorithm is able to probe the initial seconds of the expansion phase onset with high temporal resolution, $\sim 20-40$ s. Furthermore, using a network of stations, the onset of the Pil/2 waves can be mapped as a function of latitude and longitude in the ionosphere. This reveals a coherent pattern of the propagation of the onset of Pi1/2 waves above presubstorm background noise which has a clear ionospheric epicenter.

[36] The location of the ULF wave epicenter has been validated by comparing the ULF onset time and location with five selected substorms identified in the Frey et al. 
[2004] IMAGE-FUV substorm database and an isolated auroral brightening (or pseudobreakup). In each case the ULF wave onset is observed prior to optical onset as defined in the Frey substorm database, with the epicenter lying in close spatial proximity to the conjugate Northern Hemisphere Frey onset location (red crosses in Figures 6, $9 \mathrm{a}-9 \mathrm{c}, 12$, and 13) and as well as the T96 field trace of the Southern Hemisphere onset location into the Northern Hemisphere (blue crosses in Figures 6, 9a-9c, 12, and 13). In all of the substorms reported here, the global-scale auroral intensification recorded by the IMAGE-FUV instrument, and identified as substorm onset occurs $\sim 2 \mathrm{~min}$, and in one case as long as $12 \mathrm{~min}$ (though due to IMAGE tracking problems the uncertainty in the onset time is approximately $9 \mathrm{~min}$ ) after the wavelet-determined Pi1/2 onset time. Even though the FUV camera has $\sim 2 \mathrm{~min}$ cadence, the global intensification of the aurora during these substorms occurs several minutes after the first coherent and continuous observation of Pi1/2 ULF wave power above the background noise threshold. The ability to detect the initial and localized ionospheric onset of the substorm expansion phase to within $\sim 20-40 \mathrm{~s}$ is essential to understanding the sequence of events during the substorm expansion.

[37] It has been suggested [e.g., Lui, 1996; Baker et al., 1996; Angelopoulos, 2008] that the unambiguous identification of the magnetospheric processes responsible for triggering substorm expansion phase onset has been thwarted in the past due to limited spatial and temporal observations during expansion phase onset. Both in situ and ground-based observations across an extended region are required at high temporal cadence and high spatial resolution in order to resolve the causal sequence of events at substorm expansion phase onset and distinguish between competing CD and NENL models. The AWESOME technique described here offers the capability to not only provide a high temporal resolution $(\sim 20-40$ s) diagnosis of the first ionospheric signatures of substorm expansion phase onset, but also to spatially locate the epicenter which characterizes the ionospheric region of the first Pi1/2 activity. Such continent-scale magnetic Pil/2 timing and location, as provided by the AWESOME wavelet technique described here, will be crucial for interpreting in situ substorm signals, for example from the THEMIS probes, and ultimately for solving the substorm problem.

[38] Acknowledgments. This work was partially supported by a NSERC-PGSM scholarship awarded to K.R.M. and an NSERC Discovery Grant to I.R.M. CARISMA is operated by the University of Alberta and funded by the Canadian Space Agency. THEMIS is funded by NASA contract NAS5-02099. Data for this study were provided courtesy of the Canadian Magnetic Observatory System (CANMOS) network, maintained and operated by the Geological Survey of Canada; the Midcontinent Magnetoseismic Chain (McMAC), funded by the National Science foundation; and the Geophysical Institute Magnetometer Array (GIMA) at the University of Alaska, Fairbanks.

[39] Wolfgang Baumjohann thanks Mark Lester and Martin Volwerk for their assistance in evaluating this manuscript.

\section{References}

Akasofu, S. I. (1964), The development of the auroral substorm, Planet. Space Sci., 12(4), 273-282, doi:10.1016/0032-0633(64)90151-5.

Angelopoulos, V. (2008), The THEMIS mission, Space Sci. Rev., doi:10.1007/ s11214-008-9336-1, in press.

Angelopoulos, V, W. Baumjohann, C. F. Kennel, F. V. Coroniti, M. G.

Kivelson, R. Pellat, R. J. Walker, H. Lühr, and G. Paschmann (1992),
Bursty bulk flows in the Inner Central Plasma Sheet, J. Geophys. Res., 97, 4027-4039, doi:10.1029/91JA02701.

Angelopoulos, V., et al. (2008), First results from the THEMIS mission, Space Sci. Rev., doi:10.1007/s11214-008-9378-4, in press.

Baker, D. N, T. I. Pulkkinen, V. Angelopoulos, W. Baumjohann, and R. L. McPherron (1996), Neutral line model of substorms: Past results and present view, J. Geophys. Res., 101, 12,975-13,010, doi:10.1029/95JA03753.

Baumjohann, W., G. Paschmann, and C. A. Cattell (1989), Average plasma properties in the central plasma sheet, J. Geophys. Res., 94, 6597-6606, doi:10.1029/JA094iA06p06597.

Bosinger, T., et al. (1981), Correlations between PiB-type magnetic micropulsations, auroras and equivalent current structures during tow isolated substorms, J. Atmos. Terr. Phys., 43(9), 933-945, doi:10.1016/0021-9169(81)90085-4. Cramoysan, M., et al. (1995), The use of a model current wedge in the determination of the position of substorm current systems, Ann. Geophys., 13, 583-594, doi:10.1007/s00585-995-0583-0.

Daubechies, I. (1988), Orthonormal bases of compactly supported wavelets, Commun. Pure Appl. Math., 41, 909-996, doi:10.1002/cpa.3160410705. Donovan, E. F., S. Mende, B. Jackel, M. Syrjäsuo, M. Meurant, I. Voronkov, H. U. Frey, V. Angelopoulos, and M. Connors (2006), The azimuthal evolution of the substorm expansive phase onset aurora, in Proc. ICS-8, edited by M. Syrjäsuo and E. F. Donovan, pp. 55-60, Univ. of Calgary, Alberta, Canada.

Frey, H. U., and S. B. Mende (2006), Substorm onsets as observed by IMAGE-FUV, in Proc. ICS-8, edited by M. Syrjäsuo and E. F. Donovan, pp. 71-75, Univ. of Calgary, Alberta, Canada.

Frey, H. U., S. B. Mende, V. Angelopoulos, and E. F. Donovan (2004), Substorm onset observations by IMAGE-FUV, J. Geophys. Res., 109, A10304, doi:10.1029/2004JA010607.

Gelpi, C., H. J. Singer, and W. J. Hughes (1987), A comparison of magnetic signatures and DMSP auroral images at substorm onset: Three case studies, J. Geophys. Res., 92, 2447-2460, doi:10.1029/JA092iA03p02447.

Huba, J. D., N. T. Gladd, and K. Papadopoulos (1977), The lower-hybrid-drift instability as a source of anomalous resistivity for magnetic field line reconnection, Geophys. Res. Lett., 4, 125-128, doi:10.1029/GL004i003p00125. Jacobs, J. A., et al. (1964), Classification of geomagnetic micropulsations, J. Geophys. Res., 69, 180-181, doi:10.1029/JZ069i001p00180.

Kisabeth, J. L., and G. Rostoker (1977), Modelling of 3-dimensional current systems associated with magnetospheric substorms, Geophys. J. R. Astron. Soc., 49, 655-683.

Lessard, M. R., E. J. Lund, S. L. Jones, R. L. Arnoldy, J. L. Posch, M. J. Engebretson, and K. Hayashi (2006), Nature of Pi1B pulsations as inferred from ground and satellite observations, Geophys. Res. Lett., 33, L14108, doi:10.1029/2006GL026411.

Lester, M., J. W. Hughes, and H. J. Singer (1983), Polarization patterns of Pi 2 magnetic pulsations and the substorm current wedge, J. Geophys. Res., 88, 7958-7966, doi:10.1029/JA088iA10p07958.

Lester, M., H. J. Singer, D. P. Smits, and W. J. Hughes (1989), Pi 2 pulsations and the substorm current wedge: Low-latitude polarization, J. Geophys. Res., 94, 17,133-17,141, doi:10.1029/JA094iA12p17133.

Liang, J., et al. (2008), Intensification of preexisting auroral arc at substorm expansion phase onset: Wave-like disruption during the first tens of seconds, Geophys. Res. Lett., 35, L17S19, doi:10.1029/2008GL033666.

Lui, A. T. Y. (1996), Current disruption in the Earth's magnetosphere: Observations and models, J. Geophys. Res., 101, 13,067-13,088, doi:10.1029/ 96JA00079.

Lui, A. T. Y., C.-L. Chang, and P. H. Yoon (1995), Preliminary nonlocal analysis of cross-field current instability for substorm expansion onset, J. Geophys. Res., 100, 19,147-19,154, doi:10.1029/95JA01404.

Lyons, L. R. (1995), A new theory for magnetospheric substorms, J. Geophys. Res., 100, 19,069-19,081, doi:10.1029/95JA01344.

Lysak, R. L. (1988), Theory of auroral zone PiB pulsation spectra, J. Geophys. Res., 93, 5942-5946, doi:10.1029/JA093iA06p05942.

Mann, I. R., et al. (2008), The expanded and upgraded CARISMA magnetometer array, Space Sci. Rev., doi:10.1007/s11214-008-9457-6, in press.

McPherron, R. L (1972), Substorm related changes in geomagnetic tail-growth phase, Planet. Space Sci., 20, 1521-1539, doi:10.1016/00320633(72)90054-2.

Meyer, Y. (1989), Orthonormal wavelets, in Wavelets, edited by J. M. Combes, A. Grossmann, and P. Tchamitchian, pp. 21-37, Springer, Berlin.

Milling, D. K., I. J. Rae, I. R. Mann, K. R. Murphy, A. Kale, C. T. Russell, V. Angelopoulos, and S. Mende (2008), Ionospheric localisation and expansion of long-period Pil pulsations at substorm onset, Geophys. Res. Lett., 35, L17S20, doi:10.1029/2008GL033672.

Nose, M., et al. (1998), Automated detection of Pi 2 pulsations using wavelet analysis: 1 . Method and an application for substorm monitoring, Earth Planets Space, 50, 773-783.

Olson, J. V. (1999), Pi2 pulsations and substorm onsets: A review, J. Geophys. Res., 104, 17,499-17,520, doi:10.1029/1999JA900086. 
Østgaard, N., S. B. Mende, H. U. Frey, T. J. Immel, L. A. Frank, J. B. Sigwarth, and T. J. Stubbs (2004), Interplanetary magnetic field control of the location of substorm onset and auroral features in the conjugate hemispheres, J. Geophys. Res., 109, A07204, doi:10.1029/2003JA010370.

Østgaard, N., et al. (2007), Auroral conjugacy studies based on global imaging, J. Atmos. Sol. Terr. Phys., 69, 249-255, doi:10.1016/j.jastp. 2006.05.026.

Petrukovich, A. A., and A. G. Yahnin (2006), The substorm onset location controversy, Space Sci. Rev., 122, 81-87, doi:10.1007/s11214-006-7022-8.

Posch, J. L., et al. (2007), Statistical observations of spatial characteristics of Pi1B pulsations, J. Atmos. Sol. Terr. Phys., 69, 1775-1796, doi:10.1016/ j.jastp.2007.07.015.

Rae, I. J., et al. (2008), Timing and localization of ionospheric signatures associated with substorm expansion phase onset, J. Geophys. Res., 113, A00C09, doi:10.1029/2008JA013559.

Roux, A., S. Perraut, P. Robert, A. Morane, A. Pedersen, A. Korth, G. Kremser, B. Aparicio, D. Rodgers, and R. Pellinen (1991), Plasma sheet instability related to the westward travelling surge, J. Geophys. Res., 96 , 17,697-17,714, doi:10.1029/91JA01106

Russell, C. T. (1974), The solar wind and magnetospheric dynamics, in Correlated Interplanetary and Magnetospheric Observations, pp. 3-47, edited by D. E. Page, D. Reidel, Dordrecht, Netherlands.

Russell, C. T., and R. L. McPherron (1973), The magnetotail and substorms, Space Sci. Rev., 15, 205-266, doi:10.1007/BF00169321.

Russell, C. T., et al. (2008), THEMIS ground-based magnetometers, Space Sci. Rev., doi:10.1007/s11214-008-9337-0, in press.

Samson, J. C, and B. G. Harrold (1985), Characteristic time constants and velocities of high-latitude Pi2s, J. Geophys. Res., 90, 12,173-12,181, doi:10.1029/JA090iA12p12173
Shiokawa, K., W. Baumjohann, and G. Haerendel (1997), Braking of highspeed flows in the near-Earth tail, Geophys. Res. Lett., 24, 1179-1182, doi:10.1029/97GL01062

Shiokawa, K., et al. (1998), High-speed ion flow, substorm current wedge, and multiple Pi 2 pulsations, J. Geophys. Res., 103, 4491-4507, doi:10.1029/ 97JA01680

Smith, A. J., M. P. Freeman, S. Hunter, and D. K. Milling (2002), VLF, magnetic bay, and Pi2 substorm signatures at auroral and midlatitude ground stations, J. Geophys. Res., 107(A12), 1439, doi:10.1029/2002JA009389.

Tsyganenko, N. A. (1995), Modeling the Earths magnetospheric magneticfield confined within a realistic magnetopause, J. Geophys. Res., 100, 5599-5612, doi:10.1029/94JA03193.

Vasyliunas, V. M. (1970), Mathematical models of magnetospheric convection and its coupling to the ionosphere, in Particles and Fields in the Magnetosphere, pp. 60-71, edited by B. M. McCormac, D. Reidel, Norwell, Mass.

Voronkov, I. O., E. F. Donovan, and J. C. Samson (2003), Observations of the phases of the substorm, J. Geophys. Res., 108(A2), 1073, doi:10.1029/ 2002JA009314.

V. Angelopoulos and C. T. Russell, IGPP/ESS, University of California, Los Angeles, Box 951567, Los Angeles, CA 90095-1567, USA.

H. U. Frey, Space Sciences Laboratory, University of California, Berkeley, 7 Gauss Way, Berkeley, CA 94720-7450, USA.

I. R. Mann, D. K. Milling, K. R. Murphy, L. Ozeke, I. J. Rae, and C. E. J. Watt, Department of Physics, University of Alberta, Edmonton, Alberta T6G 2J1, Canada. (kmurphy@phys.ualberta.ca) 